\title{
Equilibria in Matching Markets with Soft and Hard Liquidity Constraints
}

Citation for published version (APA):

Herings, P. J-J., \& Zhou, Y. (2021). Equilibria in Matching Markets with Soft and Hard Liquidity Constraints. Maastricht University, Graduate School of Business and Economics. GSBE Research Memoranda No. 013 https://doi.org/10.26481/umagsb.2021013

Document status and date:

Published: 20/09/2021

DOI:

10.26481/umagsb.2021013

Document Version:

Publisher's PDF, also known as Version of record

\section{Please check the document version of this publication:}

- A submitted manuscript is the version of the article upon submission and before peer-review. There can be important differences between the submitted version and the official published version of record.

People interested in the research are advised to contact the author for the final version of the publication, or visit the DOI to the publisher's website.

- The final author version and the galley proof are versions of the publication after peer review.

- The final published version features the final layout of the paper including the volume, issue and page numbers.

Link to publication

\footnotetext{
General rights rights.

- You may freely distribute the URL identifying the publication in the public portal. please follow below link for the End User Agreement:

www.umlib.nl/taverne-license

Take down policy

If you believe that this document breaches copyright please contact us at:

repository@maastrichtuniversity.nl

providing details and we will investigate your claim.
}

Copyright and moral rights for the publications made accessible in the public portal are retained by the authors and/or other copyright owners and it is a condition of accessing publications that users recognise and abide by the legal requirements associated with these

- Users may download and print one copy of any publication from the public portal for the purpose of private study or research.

- You may not further distribute the material or use it for any profit-making activity or commercial gain

If the publication is distributed under the terms of Article $25 \mathrm{fa}$ of the Dutch Copyright Act, indicated by the "Taverne" license above, 


\section{Maastricht University}

P. Jean-Jacques Herings, Yu Zhou

Equilibria in Matching Markets with Soft and Hard Liquidity Constraints

$\mathrm{RM} / 21 / 013$

ISSN: $2666-8807$

\section{GSBE}

Maastricht University School of Business and Economics

Graduate School of Business and Economics

P.O Box 616

NL-6200 MD Maastricht

The Netherlands 


\title{
Equilibria in Matching Markets with Soft and Hard Liquidity Constraints*
}

\author{
P. Jean-Jacques Herings ${ }^{\dagger} \quad \mathrm{Yu}_{\mathrm{Zhou}^{\ddagger}}^{\ddagger}$
}

September 10, 2021

\begin{abstract}
We consider a matching with contracts model in the presence of liquidity constraints on the buyers side. Liquidity constraints can be either soft or hard. A convergent sequence of economies with increasingly stringent soft liquidity constraints is an economy with hard liquidity constraints at the limit. The limit of a corresponding convergent sequence of competitive equilibria may fail to be a competitive equilibrium in the limit economy. We establish limit results of two alternative notions of competitive equilibrium, quantity-constrained competitive equilibrium and expectational equilibrium, which do not suffer from such discontinuity problems. The implications of these limit results are discussed.

Keywords: Soft liquidity constraints, hard liquidity constraints, matching with contracts, competitive equilibrium, quantity-constrained competitive equilibrium, expectational equilibrium, limit result.
\end{abstract}

JEL Classification: C72, C78, D45, D52

*Yu Zhou gratefully acknowledges financial support from the Grant-in-aid for Research Activity, Japan Society for the Promotion of Science (19K13653, 20KK0027, 21H00696).

${ }^{\dagger}$ Department of Economics, Maastricht University, P.O. Box 616, 6200 MD, Maastricht, the Netherlands. E-mail: P.Herings@maastrichtuniversity.nl

${ }^{\ddagger}$ Graduate School of Economics, Kyoto University, Yoshida-Honmachi, Sakyo-ku, Kyoto, 606-8501, Japan. E-mail: zhouyu_0105@hotmail.com 


\section{Introduction}

Financial market imperfections often cause economic agents to be subject to liquidity constraints. Such constraints have important consequences for the saving behavior of consumers and investment and operational decisions by producers. ${ }^{1}$ There is also a wide range of empirical and anecdotal evidence that highlights the practical importance of buyers' limited purchasing power in auction settings. ${ }^{2}$ Understanding how liquidity constraints influence market outcomes is therefore of great importance.

Liquidity constraints come in two forms. A soft liquidity constraint refers to the case where an agent can always get additional liquidity by paying some appropriate borrowing costs. A hard liquidity constraint refers to the case where an agent is subject to a strict payment limit and cannot obtain any additional liquidity.

In matching theory, there are remarkable differences between models with soft liquidity constraints and those with hard liquidity constraints. Conditions that guarantee the existence and nice properties of competitive equilibria with soft liquidity constraints ${ }^{3}$ are no longer sufficient to tackle the models with hard constraints, in which competitive equilibria may not exist and those properties may not hold. ${ }^{4}$ Besides, these two types of liquidity constraints may also result in different monotone matching patterns among agents. ${ }^{5}$

We study the connection between economies with soft liquidity constraints and those with hard liquidity constraints. The latter is treated as the limit of economies with increasingly stringent soft liquidity constraints. We do so in a one-to-one two-sided matching with contracts model in the spirit of Crawford and Knoer (1981) and Hatfield and Milgrom (2005), extended by soft or hard liquidity constraints.

Buyers and sellers choose from a finite set of possible trades. Each trade is bilateral and designates its buyer and its seller. A contract consists of a trade and an amount of money transferred from the buyer to the seller. Buyers and sellers have general utility functions, allowing for income effects, defined on the set of contracts. At a technical level, the agents' utility functions are not continuous when going from increasingly stringent soft to hard liquidity constraints, which explains the observed anomalies.

With soft liquidity constraints, when the amount of money in a contract exceeds the buyer's initial budget, the buyer can get additional liquidity on the financial markets after paying an appropriate interest rate. A higher interest rate corresponds to a more stringent soft liquidity constraint. At the limit, when the interest rate approaches plus infinity, the

\footnotetext{
${ }^{1}$ See, e.g., Carroll (2001) and Blalock, Gertler, and Levine (2008).

${ }^{2}$ See, e.g., Che and Gale (1998).

${ }^{3}$ See, e.g., Demange and Gale (1985), Fleiner, Jagadeesan, Jankó, and Teytelboym (2019), and Schlegel (2021).

${ }^{4}$ See, e.g., Talman and Yang (2015) and Herings and Zhou (2021).

${ }^{5}$ See, e.g., Legros and Newman (2007).
} 
buyer faces a hard liquidity constraint and any monetary transfer exceeding the initial budget is not feasible.

A competitive equilibrium always exists in the case of soft liquidity constraints, but may fail to do so in the presence of hard liquidity constraints. Herings and Zhou (2021) argue that the standard notion of competitive equilibrium is not appropriate and introduce a quantity-constrained competitive equilibrium (QCCE). A QCCE extends a standard competitive equilibrium by introducing endogenously determined expectations of buyers about the availability of trades. In particular, when there is a hard liquidity constraint and the price of a trade is equal to or above this constraint, a buyer is allowed to expect no supply of the trade, and therefore demands another trade that is expected to be supplied.

Consider a sequence of economies with increasingly stringent soft liquidity constraints. We establish that a corresponding sequence of QCCE prices and outcomes always contains a convergent subsequence, and every convergent subsequence always leads to prices and outcomes that are compatible with a QCCE of the limit economy. Notice that such a result does not hold for competitive equilibria. The fact that, contrary to competitive equilibria, QCCEs behave continuously when going from increasingly stringent soft to hard liquidity constraints, reinforces the view that QCCE is the appropriate notion of competitive equilibrium.

A more fundamental notion of competitive equilibrium is expectational equilibrium as introduced in Herings (2020). The concept is formulated for general many-to-one matching models. At an expectational equilibrium, agents have endogenously determined expectations about the tradability of contracts. Agents hold expectations about the contracts that are to be supplied by agents on the other side of the market. Rationing constraints are used to express that certain contracts are not expected to be supplied. At equilibrium, for each contract at least one side is not rationed. Agents choose optimal contracts subject to the rationing constraints. Although an expectational equilibrium does not depend on prices, it coincides with a competitive equilibrium outcome when frictions like liquidity constraints are absent. We establish the limit result of expectational equilibrium outcomes when going from increasingly stringent soft to hard liquidity constraints, and conclude that expectational equilibrium does not suffer from any discontinuity problem.

We further show the equivalence between QCCE outcomes and expectational equilibrium outcomes. Together with the equivalence results in Herings and Zhou (2021), this demonstrates the coincidence of QCCE outcomes, expectational equilibrium outcomes, stable outcomes and core outcomes in our model. The limit results related to stable outcomes and core outcomes now follow as corollaries. We also argue that the limit economy with hard liquidity constraints may have equilibria that do not correspond to the limit of equilibria of economies with increasingly stringent soft liquidity constraints. 
Notions like competitive equilibrium, stable outcome, and core outcome, and their structural properties have been intensively studied in all sorts of matching models when agents have general utility functions, see, e.g., Crawford and Knoer (1981), Quinzii (1984), Demange and Gale (1985), Legros and Newman (2007), Fleiner et al. (2019), and Schlegel (2021). A matching model with general utility functions can be used to analyze matching models with soft liquidity constraints, but not to study matching models with hard liquidity constraints, which are fundamentally different.

In the assignment model with unit-demand agents and hard liquidity constraints, Talman and Yang (2015) propose an auction that constructively finds a core outcome. In one-to-one matching models with imperfectly transferable utility and unobserved heterogeneity in tastes, Galichon, Kominers, and Weber (2019) define the notion of aggregate equilibrium. In one-to-one matching models with hard liquidity constraints, Herings and Zhou (2021) propose the notion of a QCCE. They study the formation of QCCEs in decentralized markets and the structural properties of QCCEs. In many-to-one matching models with general constraints, Herings (2020) proposes expectational equilibrium and discusses its equivalence with standard notions of competitive equilibrium and stable outcomes in a large variety of settings. All these results are different from ours as they do not relate economies with hard liquidity constraints to those with increasingly stringent soft liquidity constraints.

By establishing limit results of equilibrium notions with different types of liquidity constraints, our results throw light on novel continuity properties of these equilibria. We contribute to the works mentioned above by providing a new connection between equilibria in matching models with soft liquidity constraints and those with hard liquidity constraints.

The remaining part is organized as follows. Section 2 presents the model and Section 3 studies the limit behavior of competitive equilibria and QCCEs. Section 4 extends this analysis to expectational equilibria. Section 5 discusses the relations to other solution concepts like the core and the set of stable outcomes. Section 6 contains the conclusion.

\section{The Matching Model with Liquidity Constraints}

There is a finite set of buyers $B$ and a finite set of sellers $S$. Buyers and sellers participate in bilateral trades in a finite set $\Omega$. Each trade $\omega \in \Omega$ is associated with a buyer $b(\omega) \in B$

and a seller $s(\omega) \in S$. A trade specifies the precise contractual terms of the delivery of a good or a service from the seller to the buyer, with the exception of the price against which such a transaction occurs. We denote the set of trades in $\Omega$ involving buyer $b \in B$ and 
seller $s \in S$, respectively, by

$$
\begin{aligned}
& \Omega^{b}=\{\omega \in \Omega \mid b(\omega)=b\}, \\
& \Omega^{s}=\{\omega \in \Omega \mid s(\omega)=s\} .
\end{aligned}
$$

Transactions take place by signing contracts. A contract $c=(\omega, t) \in \Omega \times \mathbb{R}$ specifies a trade $\omega \in \Omega$ and a payment $t \in \mathbb{R}$ that is transferred from buyer $b(\omega)$ to seller $s(\omega)$. For every contract $c \in \Omega \times \mathbb{R}$, let $\omega(c)$ and $t(c)$ be the corresponding trade and payment, respectively. For a set of contracts $Y \subseteq \Omega \times \mathbb{R}, \omega(Y)=\{\omega(c) \in \Omega \mid c \in Y\}$ corresponds to the set of trades related to contracts in $Y$.

We consider a one-to-one matching set-up. A buyer signs a contract with at most one seller and a seller signs a contract with at most one buyer. An agent $i \in B \cup S$ who does not sign any contract receives the no-trade option $o^{i}$.

For every buyer $b \in B$, the utility function $u^{b}:\left(\Omega^{b} \times \mathbb{R}\right) \cup\left\{o^{b}\right\} \rightarrow \mathbb{R}$ is such that, (b-i) for every $\omega \in \Omega^{b}, u^{b}(\omega, \cdot)$ is continuous and strictly decreasing in $\mathbb{R}$ and, (b-ii) for every $\omega \in \Omega^{b}$, the range of $u^{b}(\omega, \cdot)$ is all of $\mathbb{R}$. Condition (b-i) says that given a trade, a lower payment improves the buyer's utility. Condition (b-ii) says that there is no trade $\omega$ that is infinitely good or bad. That is, for every trade $\omega \in \Omega^{b}$, there is $\bar{t}_{\omega}^{b} \in \mathbb{R}$ such that $u^{b}\left(\omega, \bar{t}_{\omega}^{b}\right)=u^{b}\left(o^{b}\right)$. Moreover, for every contract $c \in \Omega^{b} \times \mathbb{R}$, for every trade $\omega^{\prime} \in \Omega^{b}$, there is $t^{\prime} \in \mathbb{R}$ such that $u^{b}(c)=u^{b}\left(\omega^{\prime}, t^{\prime}\right)$. For every $\omega \in \Omega$, we define $u^{b(\omega)}(\omega,+\infty)=-\infty$.

For every seller $s \in S$, the utility function $u^{s}:\left(\Omega^{s} \times \mathbb{R}\right) \cup\left\{o^{s}\right\} \rightarrow \mathbb{R}$ is such that, (s-i) for every $\omega \in \Omega^{s}, u^{s}(\omega, \cdot)$ is continuous and strictly increasing in $\mathbb{R}$ and, (s-ii) for every $\omega \in \Omega^{s}$, the range of $u^{s}(\omega, \cdot)$ is all of $\mathbb{R}$. The economic explanation for (s-i) and (s-ii) is analogous to (b-i) and (b-ii). Thus, for every trade $\omega \in \Omega^{s}$, there is $\underline{t}_{\omega}^{s} \in \mathbb{R}$ such that $u^{s}\left(\omega, \underline{t}_{\omega}^{s}\right)=u^{s}\left(o^{s}\right)$. Moreover, for every contract $c \in \Omega^{s} \times \mathbb{R}$, for every trade $\omega^{\prime} \in \Omega^{s}$, there is $t^{\prime} \in \mathbb{R}$ such that $u^{s}(c)=u^{s}\left(\omega^{\prime}, t^{\prime}\right)$.

Assumptions (b-ii) and (s-ii) are standard in the matching literature when agents have general utility functions, see, e.g., Demange and Gale (1985). The commonly used quasilinear utility functions satisfy (b-ii) and (s-ii).

We denote the profile of utility functions by $u=\left(u^{i}\right)_{i \in B \cup S}$.

Given a set of contracts $Y \subseteq \Omega \times \mathbb{R}, Y^{s}$ denotes the set of contracts involving seller $s \in S$ and $Y^{b}$ denotes the set of contracts involving buyer $b \in B$, so

$$
\begin{aligned}
& Y^{b}=\{c \in Y \mid b(c)=b\}, \\
& Y^{s}=\{c \in Y \mid s(c)=s\} .
\end{aligned}
$$

Notice that in case agent $i \in B \cup S$ is not part of a contract in $Y$, then $Y^{i}=\emptyset$.

The consumption set of agent $i \in B \cup S$ is equal to

$$
X^{i}=\left\{Y \subseteq \Omega^{i} \times \mathbb{R}|| Y \mid \leq 1\right\}
$$


Thus, $X^{i}$ consists of the singleton subsets of $\Omega^{i} \times \mathbb{R}$ and the empty set.

A set of contracts $A \subseteq \Omega \times \mathbb{R}$ is an outcome if, for every $i \in B \cup S, A^{i} \in X^{i}$. Let $\mathcal{A}$ be the collection of outcomes, i.e.,

$$
\mathcal{A}=\left\{A \subseteq \Omega \times \mathbb{R} \mid \text { for every } i \in B \cup S, A^{i} \in X^{i}\right\}
$$

Notice that $\emptyset \in A$, so $\emptyset$ is an outcome. More generally, for every $i \in B \cup S$, any element of $X^{i}$ is an outcome as well.

In the following, we introduce soft and hard liquidity constraints. Every buyer $b \in B$ is endowed with an amount of money $M^{b} \in \mathbb{R}_{+}$. If the payment exceeds $M^{b}$, then buyer $b$ can borrow money on the financial markets against an interest rate $r^{b} \in[0,+\infty]$. In case $r^{b} \in[0,+\infty)$, buyer $b$ faces a soft liquidity constraint. A special case of a soft liquidity constraint is the absence of a liquidity constraint if $r^{b}=0$. In case $r^{b}=+\infty$, buyer $b$ cannot make a payment exceeding $M^{b}$ and is subject to a hard liquidity constraint.

Depending on the their amount of collateral or their social network, different buyers may be able to acquire additional liquidity against different interest rates. Thus, we allow interest rates to be buyer-dependent. Let $M=\left(M^{b}\right)_{b \in B}$ be the profile of monetary endowments and $r=\left(r^{b}\right)_{b \in B}$ be the profile of interest rates.

For every buyer $b \in B$, for every contract $c \in \Omega^{b} \times \mathbb{R}$, the transfer plus borrowing cost for contract $c$ is given by

$$
t^{+}(c ; r)= \begin{cases}t(c), & \text { if } t(c) \leq M^{b} \\ t(c)+r^{b}\left(t(c)-M^{b}\right), & \text { if } t(c)>M^{b}\end{cases}
$$

The amount $t^{+}(c ; r)$ is larger than or equal to $t(c)$. In case $t(c) \leq M^{b}$, buyer $b$ does not need to borrow and $t^{+}(c ; r)=t(c)$. In case $t(c)>M^{b}$, buyer $b$ pays a borrowing cost $r^{b}\left(t(c)-M^{b}\right)$ and $t^{+}(c ; r)=t(c)+r^{b}\left(t(c)-M^{b}\right)$. If buyer $b$ faces a hard liquidity constraint, then $t(c)>M^{b}$ implies $t^{+}(c ; r)=+\infty$. By our assumptions on utility functions, such a contract is never chosen by the buyer, since it is inferior to the no-trade option.

The utility function $u^{b}$ of buyer $b \in B$ over contracts induces the utility function $U^{b}$ over outcomes. For every profile of interest rates $r \in[0,+\infty]^{B}$, for every $A \in \mathcal{A}$, in case $A^{b}=\emptyset, U^{b}(A ; r)=u^{b}\left(o^{b}\right)$, and in case $A^{b}=\{c\}, U^{b}(A ; r)=u^{b}\left(\omega(c), t^{+}(c ; r)\right)$.

The utility function $u^{s}$ of seller $s \in S$ over contracts induces the utility function $U^{s}$ over outcomes. For every $A \in \mathcal{A}$, in case $A^{s}=\emptyset, U^{s}(A)=u^{s}\left(o^{s}\right)$, and in case $A^{s}=\{c\}$, $U^{s}(A)=u^{s}(c)$.

The primitives of the economy are summarized by $\mathcal{E}=(B, S, \Omega, u, M, r)$.

Our model subsumes matching and auction models with no liquidity constraints, soft liquidity constraints, and hard liquidity constraints as special cases. For example, if there are no liquidity constraints, i.e., $r^{b}=0$ for every $b \in B$, and the set of trades $\Omega=B \times S$ 
corresponds to the possible matches between buyers and sellers, and the no-trade option to being unmatched, then our model is essentially the same as the models of Crawford and Knoer (1981) and Demange and Gale (1985). Another example given below shows that our model unifies assignment models with soft and hard liquidity constraints.

Example 2.1: The assignment model with soft liquidity constraints, e.g., Saitoh and Serizawa (Example 1, 2008), or hard liquidity constraints, e.g., Talman and Yang (2015).

There is a finite set of buyers $B$ and a finite set of sellers $S$. Each seller owns one item and is only willing to sell if the price is above a positive reserve price. Buyers want to acquire one item at most. We take the set of trades equal to $\Omega=B \times S$. For every seller $s \in S, u^{s}\left(o^{s}\right) \geq 0$ denotes the reserve price for the item in possession of seller $s$ and, for every $\omega, \omega^{\prime} \in \Omega^{s}, u^{s}(\omega, \cdot)=u^{s}\left(\omega^{\prime}, \cdot\right)$.

For every buyer $b \in B, u^{b}\left(o^{b}\right)=0$ and, for every $(\omega, t) \in \Omega^{b} \times \mathbb{R}, u^{b}(\omega, t)=V^{b}(\omega)-t$ where $V^{b}(\omega)$ is the value buyer $b$ assigns to trade $\omega$. Buyer $b \in B$ is endowed with an amount of money $M^{b} \in \mathbb{R}_{+}$. If the payment exceeds $M^{b}$, then buyer $b$ can borrow money against a market interest rate $\rho \in[0,+\infty]$, which is the same for all buyers.

For every $A \in \mathcal{A}$, for every $b \in B$, in case $A^{b}=\emptyset, U^{b}(A ; r)=u^{b}\left(o^{b}\right)=0$ and in case $A^{b}=\{(\omega, t)\}$, if buyer $b$ faces a soft liquidity constraint, i.e., $\rho \in[0,+\infty)$,

$$
U^{b}(A ; r)= \begin{cases}V^{b}(\omega)-t, & \text { if } t \leq M^{b}, \\ V^{b}(\omega)-t-\rho\left(t-M^{b}\right), & \text { if } t>M^{b},\end{cases}
$$

and if buyer $b$ faces a hard liquidity constraint, i.e., $\rho=+\infty$,

$$
U^{b}(A ; r)= \begin{cases}V^{b}(\omega)-t, & \text { if } t \leq M^{b} \\ -\infty, & \text { if } t>M^{b}\end{cases}
$$

\section{Competitive Equilibrium}

In the competitive analysis of matching models, each trade $\omega \in \Omega$ is assigned a price $p_{\omega} \in \mathbb{R}$, which results in a price vector $p \in \mathbb{R}^{\Omega}$. Prices are allowed to be personalized. For example, a seller $s \in S$ can sell an identical commodity at different prices to different buyers, something which is widely observed in online transactions. Personalized prices have been used before in competitive settings, see, e.g., Hatfield, Kominers, Nichifor, Ostrovsky, and Westkamp (2013) and Fleiner et al. (2019).

The budget set of agent $i \in B \cup S$ is given by

$$
\gamma^{i}(p)=\left\{A^{i} \in X^{i} \mid \forall c \in A^{i}, t(c)=p_{\omega(c)}\right\}, \quad p \in \mathbb{R}^{\Omega} .
$$

The budget set of agent $i$ contains those contracts in the consumption set of agent $i$ with transfers equal to prices. The budget set always contains $\emptyset$ and is therefore non-empty. 
In a competitive equilibrium, buyers take prices $p \in \mathbb{R}^{\Omega}$ as given and choose optimal contracts. The demand set of buyer $b \in B$ is given by

$$
\delta^{b}(p ; r)=\underset{A^{b} \in \gamma^{b}(p)}{\arg \max } U^{b}\left(A^{b} ; r\right), \quad p \in \mathbb{R}^{\Omega} .
$$

Sellers take prices $p \in \mathbb{R}^{\Omega}$ as given and choose optimal contracts. The demand set of seller $s \in S$ is given by

$$
\delta^{s}(p)=\underset{A^{s} \in \gamma^{s}(p)}{\arg \max } U^{s}\left(A^{s}\right), \quad p \in \mathbb{R}^{\Omega}
$$

We now define the notion of a competitive equilibrium.

Definition 3.1: An element $(p, A) \in \mathbb{R}^{\Omega} \times \mathcal{A}$ is a competitive equilibrium for the economy $\mathcal{E}=(B, S, \Omega, u, M, r)$ if:

(i) For every $b \in B, A^{b} \in \delta^{b}(p ; r)$.

(ii) For every $s \in S, A^{s} \in \delta^{s}(p)$.

In a competitive equilibrium, agents receive their demanded contracts. The usual condition that demand equals supply is implicitly incorporated in the definition of an outcome.

Crawford and Knoer (1981) show the existence of a competitive equilibrium in the absence of liquidity constraints.

Proposition 3.2: Let $\mathcal{E}=(B, S, \Omega, u, M, r)$ be an economy such that, for every $b \in B$, $r^{b}=0$. A competitive equilibrium exists.

Proposition 3.2 concerns economies without liquidity constraints. The next result deals with arbitrary soft liquidity constraints.

Proposition 3.3: Let $\mathcal{E}=(B, S, \Omega, u, M, r)$ be an economy such that $r \in[0,+\infty)^{B}$. A competitive equilibrium exists.

Proof: For every $b \in B$, we define $\widetilde{u}^{b}:\left(\Omega^{b} \times \mathbb{R}\right) \cup\left\{o^{b}\right\} \rightarrow \mathbb{R}$ by $\widetilde{u}^{b}\left(o^{b}\right)=u^{b}\left(o^{b}\right)$ and, for every $c \in \Omega^{b} \times \mathbb{R}, \widetilde{u}^{b}(c)=u^{b}\left(\omega(c), t^{+}(c ; r)\right)=U^{b}(\{c\} ; r)$. Since $r \in[0,+\infty)^{\Omega}$, it is easy to see that $\widetilde{u}^{b}$ satisfies (b-i) and (b-ii). Let $\widetilde{u}=\left(\left(\widetilde{u}^{b}\right)_{b \in B},\left(u^{s}\right)_{s \in S}\right)$.

Now consider the economy without liquidity constraints $\widetilde{\mathcal{E}}=(B, S, \Omega, \widetilde{u}, M, \widetilde{r})$, where, for every $b \in B, \widetilde{r}^{b}=0$. The utility function $\widetilde{u}^{b}$ of buyer $b \in B$ over contracts induces the utility function $\widetilde{U}^{b}$ over outcomes. By construction of $\widetilde{u}^{b}$, in case $A^{b}=\emptyset, \widetilde{U}^{b}\left(A^{b} ; \widetilde{r}\right)=$ $\widetilde{u}^{b}\left(o^{b}\right)=u^{b}\left(o^{b}\right)=U^{b}\left(A^{b} ; r\right)$, and in case $A^{b}=\{c\}, \widetilde{U}^{b}(\{c\} ; \widetilde{r})=\widetilde{u}^{b}(c)=U^{b}(\{c\} ; r)$. Thus, for every $b \in B$, for every $p \in \mathbb{R}^{\Omega}$, it holds that $\widetilde{\delta}^{b}(p ; \widetilde{r})=\delta^{b}(p ; r)$. 
We conclude from the above paragraph that the competitive equilibria of $\widetilde{\mathcal{E}}$ coincide with those of $\mathcal{E}$. By Proposition 3.2, there is a competitive equilibrium of $\widetilde{\mathcal{E}}$ and so $\mathcal{E}$ has a competitive equilibrium.

Q.E.D.

In the proof of Proposition 3.3 an economy with soft liquidity constraints is transformed into an economy without liquidity constraints in such a way that competitive equilibria in the economy without liquidity constraints coincide with the competitive equilibria in the original economy. Equilibrium existence in the original economy then follows from Proposition 3.2. Example 3.4 illustrates Proposition 3.3.

Example 3.4: Consider the economy in Example 2.1 in the presence of soft liquidity constraints with common interest rate $\rho \in[0,+\infty)$.

Take $B=\left\{b_{1}, b_{2}, b_{3}\right\}, S=\left\{s_{1}, s_{2}\right\}$, and $\Omega=\left\{\omega_{11}, \omega_{22}, \omega_{31}, \omega_{32}\right\}$, where buyer $b_{1}$ trades $\omega_{11}$ with $s_{1}$, buyer $b_{2}$ trades $\omega_{22}$ with $s_{2}$, and buyer $b_{3}$ trades $\omega_{31}$ with seller $s_{1}$ and $\omega_{32}$ with seller $s_{2}$. All agents have utility functions as described in Example 2.1. For every agent $i \in B \cup S, u^{i}\left(o^{i}\right)=0$. Buyers' monetary endowments are as follows: $M^{b_{1}}=2, M^{b_{2}}=3$, and $M^{b_{3}}=10$. Let $V^{b_{1}}\left(\omega_{11}\right)=5, V^{b_{2}}\left(\omega_{22}\right)=4, V^{b_{3}}\left(\omega_{31}\right)=6$, and $V^{b_{3}}\left(\omega_{32}\right)=7$.

We define $(p, A) \in \mathbb{R}^{\Omega} \times \mathcal{A}$ by

$$
\begin{aligned}
p_{\omega_{11}} & =p_{\omega_{31}}=\frac{3+2 \rho}{1+\rho}, \\
p_{\omega_{22}} & =p_{\omega_{32}}=\frac{4+3 \rho}{1+\rho}, \\
A & =\left\{\left(\omega_{11}, p_{\omega_{11}}\right),\left(\omega_{32}, p_{\omega_{32}}\right)\right\} .
\end{aligned}
$$

We show that $(p, A)$ satisfies Conditions (i) and (ii) of Definition 3.1 and conclude that it is a competitive equilibrium.

First, $(p, A)$ satisfies Condition (i).

For buyer $b_{1}$ it holds that $p_{\omega_{11}}>2=M^{b_{1}}$. Since

$$
t^{+}\left(\left(\omega_{11}, p_{\omega_{11}}\right) ; \rho\right)=p_{\omega_{11}}+\rho\left(p_{\omega_{11}}-M^{b_{1}}\right)=\frac{3+2 \rho}{1+\rho}+\rho\left(\frac{3+2 \rho}{1+\rho}-2\right)=3<5=V^{b_{1}}\left(\omega_{11}\right),
$$

buyer $b_{1}$ strictly prefers buying $\omega_{11}$ at price $p_{\omega_{11}}$ over the no-trade option. Thus $A^{b_{1}}=$ $\left\{\left(\omega_{11}, p_{\omega_{11}}\right)\right\} \in \delta^{b_{1}}(p ; \rho)=\left\{\left\{\left(\omega_{11}, p_{\omega_{11}}\right)\right\}\right\}$.

For buyer $b_{2}$ it holds that $p_{\omega_{22}}>3=M^{b_{2}}$. Since

$$
t^{+}\left(\left(\omega_{32}, p_{\omega_{32}}\right) ; \rho\right)=p_{\omega_{22}}+\rho\left(p_{\omega_{22}}-M^{b_{2}}\right)=\frac{4+3 \rho}{1+\rho}+\rho\left(\frac{4+3 \rho}{1+\rho}-3\right)=4=V^{b_{2}}\left(\omega_{22}\right),
$$

buyer $b_{2}$ is indifferent between acquiring $\omega_{22}$ against price $p_{\omega_{22}}$ and not trading. Thus $A^{b_{2}}=\emptyset \in \delta^{b_{2}}(p ; \rho)=\left\{\emptyset,\left\{\left(\omega_{22}, p_{\omega_{22}}\right)\right\}\right\}$.

For buyer $b_{3}$ it holds that $p_{\omega_{31}}, p_{\omega_{32}}<10=M^{b_{3}}$. Since $6-p_{\omega_{31}}=7-p_{\omega_{32}}>0$, at prices $p_{\omega_{31}}$ and $p_{\omega_{32}}$, buyer $b_{3}$ is indifferent between $\omega_{31}$ and $\omega_{32}$ and strictly prefers 
both trades to the no-trade option. We find that $A^{b_{3}}=\left\{\left(\omega_{32}, p_{\omega_{32}}\right)\right\} \in \delta^{b_{3}}(p ; \rho)=$ $\left\{\left\{\left(\omega_{31}, p_{\omega_{31}}\right)\right\},\left\{\left(\omega_{32}, p_{\omega_{32}}\right)\right\}\right\}$.

Second, $(p, A)$ satisfies Condition (ii). Since $p_{\omega_{11}}=p_{\omega_{31}}>0$ and $p_{\omega_{22}}=p_{\omega_{32}}>0$, seller $s_{1}$ is willing to supply either $\omega_{11}$ or $\omega_{31}$ and seller $s_{2}$ is willing to supply either $\omega_{22}$ or $\omega_{32}$. We conclude that

$$
\begin{aligned}
& A^{s_{1}}=\left\{\left(\omega_{11}, p_{\omega_{11}}\right)\right\} \in \delta^{s_{1}}(p)=\left\{\left\{\left(\omega_{11}, p_{\omega_{11}}\right)\right\},\left\{\left(\omega_{31}, p_{\omega_{31}}\right)\right\}\right\}, \\
& A^{s_{2}}=\left\{\left(\omega_{32}, p_{\omega_{32}}\right)\right\} \in \delta^{s_{2}}(p)=\left\{\left\{\left(\omega_{22}, p_{\omega_{22}}\right)\right\},\left\{\left(\omega_{32}, p_{\omega_{32}}\right)\right\}\right\} .
\end{aligned}
$$

We next consider the transition from soft to hard liquidity constraints. It turns out that the buyers' utility functions over outcomes display discontinuities, which leads to the non-existence of competitive equilibria. Example 3.5 illustrates this point.

Example 3.5: We consider the economy of Example 3.4 in the presence of hard liquidity constraints, which can be modeled by setting the common interest rate $\rho=+\infty$. The utility function of buyer $b_{1}$ over outcomes exhibits a discontinuity at a price equal to monetary endowment $M^{b_{1}}=2$ : For every $t \leq 2$ it holds that $U^{b_{1}}\left(\left\{\left(\omega_{11}, t\right)\right\} ; \rho\right)=V^{b_{1}}\left(\omega_{11}\right)-t=5-t \geq$ 3 and for every $t>2$ we have $U^{b_{1}}\left(\left\{\left(\omega_{11}, t\right)\right\} ; \rho\right)=-\infty$. The utility functions of buyers $b_{2}$ and $b_{3}$ display similar discontinuities at prices equal to their monetary endowments.

We argue that there is no competitive equilibrium. By contradiction, suppose there is a competitive equilibrium $(p, A)$. Since each buyer can trade with at most one seller, there is at least one buyer who does not trade. In case buyer $b_{1}$ chooses the no-trade option, it holds that $p_{\omega_{11}}>2$. Seller $s_{1}$ can trade with buyers $b_{1}$ and $b_{3}$. Since $b_{1}$ chooses the no-trade option, $s_{1}$ must trade with buyer $b_{3}$ at a price $p_{\omega_{31}} \geq p_{\omega_{11}}>2$. This implies that $p_{\omega_{32}} \geq p_{\omega_{31}}+1>3$, since otherwise buyer $b_{3}$ is not willing to trade with $s_{1}$. Seller $s_{2}$ can trade with buyers $b_{2}$ and $b_{3}$. Since $b_{3}$ trades with $s_{1}$, seller $s_{2}$ must trade with buyer $b_{2}$ at a price $p_{\omega_{22}} \geq p_{\omega_{32}}>3$. Since $p_{\omega_{22}}$ exceeds the monetary endowment of buyer $b_{2}$ and $\rho=+\infty$, this leads to a contradiction.

In case buyer $b_{2}$ does not trade or buyer $b_{3}$ does not trade, similar contradictions can be derived. Thus, there is no competitive equilibrium.

Herings and Zhou (2021) study models with hard liquidity constraints and propose the notion of a quantity-constrained competitive equilibrium. Buyers form expectations about the availability of trades. A buyer may expect that a trade is not available or, equivalently, a buyer may expect a binding quantity constraint, if the buyer faces a hard liquidity constraint and the price of the trade is equal to or above the buyer's monetary endowment. The reason is that these are the only circumstances under which it is impossible for the 
buyer to offer a higher transfer to the seller in case the seller makes another trade. For every $\omega \in \Omega$, let $q_{\omega} \in\{0,1\}$ be the quantity constraint of trade $\omega$, where $q_{\omega}=1$ means that buyer $b(\omega)$ expects trade $\omega$ to be available and $q_{\omega}=0$ means that buyer $b(\omega)$ expects trade $\omega$ not to be available. We denote the vector of quantity constraints by $q=\left(q_{\omega}\right)_{\omega \in \Omega}$.

The constrained budget set of buyer $b \in B$ is given by

$$
\gamma^{b}(p, q)=\left\{A^{b} \in X^{b} \mid \forall c \in A^{b}, t(c)=p_{\omega(c)} \text { and } q_{\omega(c)}=1\right\},(p, q) \in \mathbb{R}^{\Omega} \times\{0,1\}^{\Omega} .
$$

The constrained budget set of buyer $b$ contains those contracts that involve buyer $b$, i.e., $\{c\} \in X^{b}$, buyer $b$ expects them to be supplied, i.e., $q_{\omega(c)}=1$, and the payment is equal to the price, i.e., $t(c)=p_{\omega(c)}$. The set $\gamma^{b}(p, q)$ always contains $\emptyset$ and is therefore non-empty.

The constrained demand set of buyer $b \in B$ is given by

$$
\delta^{b}(p, q ; r)=\underset{A^{b} \in \gamma^{b}(p, q)}{\arg \max } U^{b}\left(A^{b} ; r\right), \quad(p, q) \in \mathbb{R}^{\Omega} \times\{0,1\}^{\Omega} .
$$

The constrained demand set of buyer $b$ equals the set of contracts which maximize buyer $b$ 's utility over the contracts in the constrained budget set.

Since a seller does not face liquidity constraints, the seller's decision problem is the same as before. A quantity-constrained competitive equilibrium is defined as follows.

Definition 3.6: An element $(p, q, A) \in \mathbb{R}^{\Omega} \times\{0,1\}^{\Omega} \times \mathcal{A}$ is a quantity-constrained competitive equilibrium ( $Q C C E$ ) for the economy $\mathcal{E}=(B, S, \Omega, u, M, r)$ if:

(i) For every $b \in B, A^{b} \in \delta^{b}(p, q ; r)$.

(ii) For every $s \in S, A^{s} \in \delta^{s}(p)$.

(iii) For every $\omega \in \Omega$, if $q_{\omega}=0$, then $r^{b(\omega)}=+\infty$ and $p_{\omega} \geq M^{b(\omega)}$.

The first two conditions of Definition 3.6 correspond to optimization by buyers that take $p$ and $q$ as given and optimization by sellers that take $p$ as given. These two conditions also imply equality of demand and supply at a QCCE. The third condition reflects that when buyer $b(\omega)$ expects trade $\omega$ not to be supplied, then buyer $b(\omega)$ faces a hard liquidity constraint, i.e., $r^{b(\omega)}=+\infty$, and the price is larger than or equal to the buyer's monetary endowment, i.e., $p_{\omega} \geq M^{b(\omega)}$. Since this condition only applies in case of a hard liquidity constraint, a QCCE coincides with a competitive equilibrium for models with soft liquidity constraints. Both $p$ and $q$ are endogenously determined in a QCCE.

QCCE is an equilibrium concept that is rooted in general equilibrium theory. Buyers form expectations about prices and quantity constraints and optimize accordingly. They do not need to form expectations about the behavior of other buyers and sellers. Since sellers do not face liquidity constraints, their decision problem is the usual one, coinciding with the one used in the definition of a competitive equilibrium. Prices and quantity constraints 
are in equilibrium if optimization by buyers and sellers leads to equality of supply and demand, i.e., markets clear.

In Definition 3.6, if for every $\omega \in \Omega, q_{\omega}=1$, then a QCCE reduces to a competitive equilibrium, also in the presence of hard liquidity constraints.

Example 3.7 illustrates QCCE.

Example 3.7: Consider the economy with hard liquidity constraints in Example 3.5. As argued before, it has no competitive equilibrium. We illustrate that it has QCCEs.

Let $(\bar{p}, \bar{q}, \bar{A}) \in \mathbb{R}^{\Omega} \times\{0,1\}^{\Omega} \times \mathcal{A}$ be defined by

$$
\begin{aligned}
& \bar{p}=\left(\bar{p}_{\omega_{11}}, \bar{p}_{\omega_{22}}, \bar{p}_{\omega_{31}}, \bar{p}_{\omega_{32}}\right)=(2,3,2,3), \\
& \bar{q}=\left(\bar{q}_{\omega_{11}}, \bar{q}_{\omega_{22}}, \bar{q}_{\omega_{31}}, \bar{q}_{\omega_{32}}\right)=(1,0,1,1), \\
& \bar{A}=\left\{\left(\omega_{11}, 2\right),\left(\omega_{32}, 3\right)\right\} .
\end{aligned}
$$

We show that $(\bar{p}, \bar{q}, \bar{A})$ is a QCCE by verifying that it satisfies Conditions (i), (ii), and (iii) of Definition 3.6.

First, $(\bar{p}, \bar{q}, \bar{A})$ satisfies Condition (i).

For buyer $b_{1}$, it holds that $\gamma^{b_{1}}(\bar{p}, \bar{q})=\left\{\emptyset,\left\{\left(\omega_{11}, 2\right)\right\}\right\}$ and $\bar{A}^{b_{1}}=\left\{\left(\omega_{11}, 2\right)\right\} \in \delta^{b_{1}}(\bar{p}, \bar{q} ; \rho)=$ $\left\{\left\{\left(\omega_{11}, 2\right)\right\}\right\}$ For buyer $b_{2}$, we have $\gamma^{b_{2}}(\bar{p}, \bar{q})=\{\emptyset\}$ and so $\bar{A}^{b_{2}}=\emptyset \in \delta^{b_{2}}(\bar{p}, \bar{q} ; \rho)=\{\emptyset\}$. For buyer $b_{3}$, it holds that $\gamma^{b_{3}}(\bar{p}, \bar{q})=\left\{\emptyset,\left\{\left(\omega_{31}, 2\right)\right\},\left\{\left(\omega_{32}, 3\right)\right\}\right\}$ and so $\bar{A}^{b_{3}}=\left\{\left(\omega_{32}, 3\right)\right\} \in$ $\delta^{b_{3}}(\bar{p}, \bar{q} ; \rho)=\left\{\left\{\left(\omega_{31}, 2\right)\right\},\left\{\left(\omega_{32}, 3\right)\right\}\right\}$.

Second, $(\bar{p}, \bar{q}, \bar{A})$ satisfies Condition (ii).

Since all trades involving seller $s_{1}$ have the same price, it is easy to see that $\bar{A}^{s_{1}}=$ $\left\{\left(\omega_{11}, 2\right)\right\} \in \delta^{s_{1}}(\bar{p})=\left\{\left\{\left(\omega_{11}, 2\right)\right\},\left\{\left(\omega_{31}, 2\right)\right\}\right\}$. The same argument applies to seller $s_{2}$, so $\bar{A}^{s_{2}}=\left\{\left(\omega_{32}, 3\right)\right\} \in \delta^{s_{2}}(\bar{p})=\left\{\left\{\left(\omega_{22}, 3\right)\right\},\left\{\left(\omega_{32}, 3\right)\right\}\right\}$.

A quantity constraint is expected for trade $\omega_{22}$ by $b_{2}$, who is subject to a hard financial constraint and has a monetary endowment equal to $p_{\omega_{22}}$. It follows that $(\bar{p}, \bar{q}, \bar{A})$ satisfies Condition (iii).

A similar argument shows that $\left(\bar{p}, q^{\prime}, A^{\prime}\right)$ such that $q^{\prime}=\left(q_{\omega_{11}}^{\prime}, q_{\omega_{22}}^{\prime}, q_{\omega_{31}}^{\prime}, q_{\omega_{32}}^{\prime}\right)=(0,1,1,1)$ and $A^{\prime}=\left\{\left(\omega_{22}, 3\right),\left(\omega_{31}, 2\right)\right\}$ is a QCCE.

Herings and Zhou (2021) study matching models with hard liquidity constraints and show the existence of QCCEs. Although they do not explicitly study models with soft liquidity constraints, these models are contained as special cases when the same transformation is used as in the proof of Proposition 3.3. Thus, we have the following result.

Proposition 3.8: Let $\mathcal{E}=(B, S, \Omega, u, M, r)$ be an economy such that $r \in[0,+\infty]^{B}$. A QCCE exists. 
In an economy with hard liquidity constraints, and perhaps some agents with soft liquidity constraints, a competitive equilibrium may fail to exist, but a QCCE always exists. In an economy with only soft liquidity constraints, competitive equilibria are equivalent to QCCEs. An immediate implication is therefore that Propositions 3.2 and Proposition 3.3 are special cases of Proposition 3.8.

We turn next to the analysis of an economy with hard liquidity constraints as the limit of an economy with increasingly stringent soft liquidity constraints.

The sequence of economies $\left(\mathcal{E}^{n}\right)_{n \in \mathbb{N}}=\left(B, S, \Omega, u, M, r^{n}\right)_{n \in \mathbb{N}}$ is said to converge to an economy $\overline{\mathcal{E}}=(B, S, \Omega, u, M, \bar{r})$ if $\bar{r}=\lim _{n \rightarrow \infty} r^{n}$. A sequence of outcomes $\left(A^{n}\right)_{n \in \mathbb{N}}$ in $\mathcal{A}$ is said to converge to an outcome $A \in \mathcal{A}$ if there is $n^{\prime} \in \mathbb{N}$ such that, for every $n \geq n^{\prime}$, $\omega\left(A^{n}\right)=\omega(A)$ and, for every $(\omega, t) \in A$, for every $c^{n} \in A^{n}$ with $\omega\left(c^{n}\right)=\omega, \lim _{n \rightarrow \infty} t\left(c^{n}\right)=$ t. A sequence of equilibria, e.g., competitive equilibria or QCCEs, is convergent if the sequence of corresponding equilibrium prices and outcomes is convergent.

Since utility functions over outcomes exhibit discontinuities in the presence of hard liquidity constraints, it is not a priori clear whether the limit of a sequence of equilibria is an equilibrium of the limit economy. Since economies with hard liquidity constraints may not possess competitive equilibria, the best one can hope for is that the limit of a sequence of equilibria is a QCCE. We establish the following general limit result.

Theorem 3.9: Let $\left(\mathcal{E}^{n}\right)_{n \in \mathbb{N}}$ be a sequence of economies that converges to an economy $\overline{\mathcal{E}}$. For every $n \in \mathbb{N}$, let $\left(p^{n}, q^{n}, A^{n}\right)$ be a QCCE of the economy $\mathcal{E}^{n}$.

(i) The sequence $\left(p^{n}, A^{n}\right)_{n \in \mathbb{N}}$ has a convergent subsequence.

(ii) Let $(\bar{p}, \bar{A})$ be the limit of a convergent subsequence of $\left(p^{n}, A^{n}\right)_{n \in \mathbb{N}}$. Then there is $\bar{q} \in$ $\{0,1\}^{\Omega}$ such that $(\bar{p}, \bar{q}, \bar{A})$ is a QCCE of $\overline{\mathcal{E}}$.

\section{Proof:}

Part (i): Since the set of trades is finite, there is a subsequence $\left(A^{n_{m}}\right)_{m \in \mathbb{N}}$ of $\left(A^{n}\right)_{n \in \mathbb{N}}$ such that the set of trades $\omega\left(A^{n_{m}}\right)$ does not depend on $n_{m}$. Define $\bar{\Omega}=\omega\left(A^{n_{m}}\right)$.

Step 1: For every $\omega \in \bar{\Omega}$, the sequence $\left(p_{\omega}^{n_{m}}\right)_{m \in \mathbb{N}}$ is bounded.

Fix some $m \in \mathbb{N}$. Let $\omega \in \bar{\Omega}, b=b(\omega)$, and $s=s(\omega)$. Since $\left(p^{n_{m}}, q^{n_{m}}, A^{n_{m}}\right)$ is a QCCE of $\mathcal{E}^{n_{m}}$, it holds that $U^{s}\left(A^{n_{m}}\right)=u^{s}\left(\omega, p_{\omega}^{n_{m}}\right) \geq u^{s}\left(o^{s}\right)=u^{s}\left(\omega, \underline{t}_{\omega}^{s}\right)$. It follows that $\underline{t}_{\omega}^{s} \leq p_{\omega}^{n_{m}}$.

There is $t_{\omega}^{n_{m}} \geq p_{\omega}^{n_{m}}$ such that $u^{b}\left(\omega, t_{\omega}^{n_{m}}\right)=U^{b}\left(\left\{\left(\omega, p_{\omega}^{n_{m}}\right)\right\} ; r^{n_{m}}\right)$. Since $\left(p^{n_{m}}, q^{n_{m}}, A^{n_{m}}\right)$ is a QCCE of $\mathcal{E}^{n_{m}}$, it holds that $u^{b}\left(\omega, t_{\omega}^{n_{m}}\right)=U^{b}\left(\left\{\left(\omega, p_{\omega}^{n_{m}}\right)\right\} ; r^{n_{m}}\right) \geq u^{b}\left(o^{b}\right)=u^{b}\left(\omega, \bar{t}_{\omega}^{b}\right)$. It follows that $p_{\omega}^{n_{m}} \leq t_{\omega}^{n_{m}} \leq \bar{t}_{\omega}^{b}$.

We conclude that, for every $m \in \mathbb{N}, \underline{t}_{\omega}^{s} \leq p_{\omega}^{n_{m}} \leq \bar{t}_{\omega}^{b}$.

Step 2: For every $\omega \in \Omega \backslash \bar{\Omega}$, the sequence $\left(p_{\omega}^{n_{m}}\right)_{m \in \mathbb{N}}$ is bounded.

Fix some $m \in \mathbb{N}$. Let $\omega \in \Omega \backslash \bar{\Omega}, b=b(\omega)$, and $s=s(\omega)$.

Assume $\left(A^{n_{m}}\right)^{b}=\emptyset$. Consider the case where $p_{\omega}^{n_{m}}<M^{b}$, so $q_{\omega}^{n_{m}}=1$. Since $\min \left\{0, \bar{t}_{\omega}^{b}\right\} \leq$ 
$M^{b}$ and $\left(p^{n_{m}}, q^{n_{m}}, A^{n_{m}}\right)$ is a QCCE of $\mathcal{E}^{n_{m}}$, it holds that

$$
\begin{aligned}
U^{b}\left(\left\{\omega, \min \left\{0, \bar{t}_{\omega}^{b}\right\}\right\} ; r^{n_{m}}\right) & =u^{b}\left(\omega, \min \left\{0, \bar{t}_{\omega}^{b}\right\}\right) \geq u^{b}\left(\omega, \bar{t}_{\omega}^{b}\right)=u^{b}\left(o^{b}\right)=U^{b}\left(A^{n_{m}} ; r^{n_{m}}\right) \\
& \geq U^{b}\left(\left\{\omega, p_{\omega}^{n_{m}}\right\} ; r^{n_{m}}\right),
\end{aligned}
$$

and so $p_{\omega}^{n_{m}} \geq \min \left\{0, \bar{t}_{\omega}^{b}\right\}$. In case $p_{\omega}^{n_{m}} \geq M^{b} \geq 0$, it also holds that $p_{\omega}^{n_{m}} \geq \min \left\{0, \bar{t}_{\omega}^{b}\right\}$.

Assume $\left(A^{n_{m}}\right)^{b} \neq \emptyset$. Consider the case where $p_{\omega}^{n_{m}}<M^{b}$, so $q_{\omega}^{n_{m}}=1$. Let $\left(A^{n_{m}}\right)^{b}=$ $\left\{\left(\omega^{\prime}, p_{\omega^{\prime}}^{n_{m}}\right)\right\}$ and $s\left(\omega^{\prime}\right)=s^{\prime}$. Since $\omega^{\prime} \in \bar{\Omega}$, by Step 1, it holds that $\underline{t}_{\omega^{\prime}}^{s^{\prime}} \leq p_{\omega^{\prime}}^{n_{m}} \leq \bar{t}_{\omega^{\prime}}^{b}$. Let $t_{\omega}^{b} \in \mathbb{R}$ be such that $u^{b}\left(\omega^{\prime}, \underline{t}_{\omega^{\prime}}^{s^{\prime}}\right)=u^{b}\left(\omega, t_{\omega}^{b}\right)$. Since $\underline{t}_{\omega^{\prime}}^{s^{\prime}} \leq p_{\omega^{\prime}}^{n_{m}}, \min \left\{0, t_{\omega}^{b}\right\} \leq M^{b}$, and $\left(p^{n_{m}}, q^{n_{m}}, A^{n_{m}}\right)$ is a QCCE of $\mathcal{E}^{n_{m}}$, it holds that

$$
\begin{aligned}
U^{b}\left(\left\{\omega, \min \left\{0, t_{\omega}^{b}\right\}\right\} ; r^{n_{m}}\right) & =u^{b}\left(\omega, \min \left\{0, t_{\omega}^{b}\right\}\right) \geq u^{b}\left(\omega^{\prime}, \underline{t}_{\omega^{\prime}}^{s^{\prime}}\right) \geq u^{b}\left(\omega^{\prime}, p_{\omega^{\prime}}^{n_{m}}\right) \\
& \geq U^{b}\left(A^{n_{m}} ; r^{n_{m}}\right) \geq U^{b}\left(\left\{\omega, p_{\omega}^{n_{m}}\right\} ; r^{n_{m}}\right),
\end{aligned}
$$

and so $p_{\omega}^{n_{m}} \geq \min \left\{0, t_{\omega}^{b}\right\}$. In case $p_{\omega}^{n_{m}} \geq M^{b} \geq 0$, it also holds that $p_{\omega}^{n_{m}} \geq \min \left\{0, t_{\omega}^{b}\right\}$.

Assume $\left(A^{n_{m}}\right)^{s}=\emptyset$. Since $\left(p^{n_{m}}, q^{n_{m}}, A^{n_{m}}\right)$ is a QCCE of $\mathcal{E}^{n_{m}}$, it holds that $U^{s}\left(A^{n_{m}}\right)=$ $u^{s}\left(o^{s}\right)=u^{s}\left(\omega, \underline{t}_{\omega}^{s}\right) \geq u^{s}\left(\omega, p_{\omega}^{n_{m}}\right)$. It follows that $p_{\omega}^{n_{m}} \leq \underline{t}_{\omega}^{s}$.

Assume $\left(A^{n_{m}}\right)^{s} \neq \emptyset$. Let $\left(A^{n_{m}}\right)^{s}=\left\{\left(\omega^{\prime}, p_{\omega^{\prime}}^{n_{m}}\right)\right\}$ and $b\left(\omega^{\prime}\right)=b^{\prime}$. Since $\omega^{\prime} \in \bar{\Omega}$, by Step 1, it holds that $\underline{t}_{\omega^{\prime}}^{s} \leq p_{\omega^{\prime}}^{n_{m}} \leq \bar{t}_{\omega^{\prime}}^{b^{\prime}}$. By (s-ii), there is $t_{\omega}^{s} \in \mathbb{R}$ such that $u^{s}\left(\omega^{\prime}, \bar{t}_{\omega^{\prime}}^{b^{\prime}}\right)=u^{s}\left(\omega, t_{\omega}^{s}\right)$. Since $\left(p^{n_{m}}, q^{n_{m}}, A^{n_{m}}\right)$ is a QCCE of $\mathcal{E}^{n_{m}}$, it holds that

$$
U^{s}\left(\left\{\omega, t_{\omega}^{s}\right\}\right)=U^{s}\left(\left\{\omega^{\prime}, \bar{t}_{\omega^{\prime}}^{b^{\prime}}\right\}\right) \geq U^{s}\left(\left\{\omega^{\prime}, p_{\omega^{\prime}}^{n_{m}}\right\}\right)=U^{s}\left(A^{n_{m}}\right) \geq U^{s}\left(\left\{\omega, p_{\omega}^{n_{m}}\right\}\right),
$$

and so $p_{\omega}^{n_{m}} \leq t_{\omega}^{s}$.

Consequently, Step 2 holds.

By Steps 1 and 2, it follows that the sequence $\left(p^{n}, A^{n}\right)_{n \in \mathbb{N}}$ has a convergent subsequence. Part (ii): Without loss of generality, assume the sequence $\left(p^{n}, A^{n}\right)_{n \in \mathbb{N}}$ is convergent and has limit $(\bar{p}, \bar{A})$. Define $\bar{\Omega}=\omega(\bar{A})$.

Let $\bar{q} \in\{0,1\}^{\Omega}$ be such that (i) for every $\omega \in \bar{\Omega}, \bar{q}_{\omega}=1$, and (ii), for every $\omega \in \Omega \backslash \bar{\Omega}$, if $r^{b(\omega)}=+\infty$ and $\bar{p}_{\omega} \geq M^{b(\omega)}, \bar{q}_{\omega}=0$, and if $r^{b(\omega)}<+\infty$ or $\bar{p}_{\omega}<M^{b(\omega)}, \bar{q}_{\omega}=1$. Since for every $n \in \mathbb{N}, A^{n}$ is a QCCE outcome and the sequence $\left(A^{n}\right)_{n \in \mathbb{N}}$ converges to $\bar{A}$, it is easily seen that $\bar{A}$ is an outcome. We show next that $(\bar{p}, \bar{q}, \bar{A})$ is a QCCE of the limit economy $\overline{\mathcal{E}}$. Step 1: Condition (i) of Definition 3.6 holds.

By contradiction, suppose that there is a buyer $b \in B$ such that $\bar{A}^{b} \notin \delta^{b}(\bar{p}, \bar{q} ; \bar{r})$.

Case 1: $\bar{A}^{b}=\emptyset$.

Since $\bar{A}^{b}=\emptyset \notin \delta^{b}(\bar{p}, \bar{q} ; \bar{r})$, there is $\omega^{\prime} \in \Omega^{b}$ such that $\left\{\left(\omega^{\prime}, \bar{p}_{\omega^{\prime}}\right)\right\} \in \delta^{b}(\bar{p}, \bar{q} ; \bar{r}), U^{b}(\emptyset ; \bar{r})<$ $U^{b}\left(\left\{\left(\omega^{\prime}, \bar{p}_{\omega^{\prime}}\right)\right\} ; \bar{r}\right)$, and $\bar{q}_{\omega^{\prime}}=1$. If $\bar{r}^{b}=+\infty$, it holds by the construction of $\bar{q}$ that $\bar{p}_{\omega^{\prime}}<M^{b}$. For sufficiently large $n$, we have that $p_{\omega^{\prime}}^{n}<M^{b}, q_{\omega^{\prime}}^{n}=1$, and $U^{b}\left(\emptyset ; r^{n}\right)=$ $u^{b}\left(o^{b}\right)<u^{b}\left(\omega^{\prime}, p_{\omega^{\prime}}^{n}\right)=U^{b}\left(\left\{\left(\omega^{\prime}, p_{\omega^{\prime}}^{n}\right)\right\} ; r^{n}\right)$, contradicting that $\left(p^{n}, q^{n}, A^{n}\right)$ is a QCCE of $\mathcal{E}^{n}$. If $\bar{r}^{b}<+\infty$, we have for sufficiently large $n$ that $U^{b}\left(\emptyset ; r^{n}\right)=u^{b}\left(o^{b}\right)<U^{b}\left(\left\{\left(\omega^{\prime}, p_{\omega^{\prime}}^{n}\right)\right\} ; r^{n}\right)$, contradicting that $\left(p^{n}, q^{n}, A^{n}\right)$ is a QCCE of $\mathcal{E}^{n}$. 
Case 2: $\bar{A}^{b} \neq \emptyset$.

Let $\omega$ be the unique element of $\omega\left(\bar{A}^{b}\right)$. First, we show that if $\bar{r}^{b}=+\infty$, then $\bar{p}_{\omega} \leq M^{b}$. By contradiction, suppose that $\bar{p}_{\omega}>M^{b}$. There is $\varepsilon>0$ such that $\bar{p}_{\omega}>M^{b}+\varepsilon$. For sufficiently large $n$, we have that $p_{\omega}^{n} \geq M^{b}+\varepsilon$ and $r^{n} \varepsilon>\bar{t}_{\omega}^{b}$. This implies that

$$
U^{b}\left(A^{n} ; r^{n}\right)=u^{b}\left(\omega, r^{n}\left(p_{\omega}^{n}-M^{b}\right)+p_{\omega}^{n}\right) \leq u^{b}\left(\omega, r^{n} \varepsilon\right)<u^{b}\left(\omega, \bar{t}_{\omega}^{b}\right)=u^{b}\left(o^{b}\right)=U^{b}\left(\emptyset ; r^{n}\right),
$$

contradicting that $\left(p^{n}, q^{n}, A^{n}\right)$ is a QCCE of $\mathcal{E}^{n}$. Consequently, we have that $\bar{p}_{\omega} \leq M^{b}$.

Since $\bar{A}^{b} \notin \delta^{b}(\bar{p}, \bar{q} ; \bar{r})$, there is $\tilde{A}^{b} \in \delta^{b}(\bar{p}, \bar{q} ; \bar{r})$ such that $U^{b}\left(\bar{A}^{b} ; \bar{r}\right)<U^{b}\left(\widetilde{A}^{b} ; \bar{r}\right)$.

Case 2-1: $\bar{r}^{b}=+\infty$.

By the analysis above, it holds that $\bar{p}_{\omega} \leq M^{b}$. For every $n \in \mathbb{N}$, we have that

$$
U^{b}\left(\left\{\left(\omega, p_{\omega}^{n}\right)\right\} ; r^{n}\right)=u^{b}\left(\omega, p_{\omega}^{n}+r^{n, b} \max \left\{0, p_{\omega}^{n}-M^{b}\right\}\right) \leq u^{b}\left(\omega, p_{\omega}^{n}\right) .
$$

Assume $\widetilde{A}^{b}=\emptyset$. Since $\lim _{n \rightarrow \infty} u^{b}\left(\omega, p_{\omega}^{n}\right)=u^{b}\left(\omega, \bar{p}_{\omega}\right)=U^{b}\left(\bar{A}^{b} ; \bar{r}\right)$, for sufficiently large $n$, we have that $U^{b}\left(\left\{\left(\omega, p_{\omega}^{n}\right)\right\} ; r^{n}\right)<U^{b}\left(\emptyset ; r^{n}\right)$, contradicting that $\left(p^{n}, q^{n}, A^{n}\right)$ is a QCCE of $\mathcal{E}^{n}$.

Assume $\widetilde{A}^{b} \neq \emptyset$. Let $\widetilde{\omega}$ be the unique element of $\omega\left(\widetilde{A}^{b}\right)$. It holds that $\bar{q}_{\widetilde{\omega}}=1$. Since $\bar{r}^{b}=+\infty$, by the construction of $\bar{q}$, it holds that $\bar{p}_{\widetilde{\omega}}<M^{b}$. Since $\lim _{n \rightarrow \infty} u^{b}\left(\omega, p_{\omega}^{n}\right)=$ $u^{b}\left(\omega, \bar{p}_{\omega}\right)=U^{b}\left(\bar{A}^{b} ; \bar{r}\right)$, it follows that $U^{b}\left(\left\{\left(\omega, p_{\omega}^{n}\right)\right\} ; r^{n}\right)<U^{b}\left(\left\{\left(\widetilde{\omega}, p_{\widetilde{\omega}}^{n}\right)\right\} ; r^{n}\right)$ for sufficiently large $n$, contradicting that $\left(p^{n}, q^{n}, A^{n}\right)$ is a QCCE of $\mathcal{E}^{n}$.

Case 2-2: $\bar{r}^{b}<+\infty$.

If $\widetilde{A}^{b}=\emptyset$, we have for $n$ sufficiently large that $U^{b}\left(\left\{\left(\omega, p_{\omega}^{n}\right)\right\} ; r^{n}\right)<U^{b}\left(\emptyset ; r^{n}\right)$, contradicting that $\left(p^{n}, q^{n}, A^{n}\right)$ is a QCCE of $\mathcal{E}^{n}$. If $\widetilde{A}^{b} \neq \emptyset$, let $\widetilde{\omega}$ be the unique element of $\omega\left(\widetilde{A}^{b}\right)$. Since $\bar{r}^{b}<+\infty$, it holds that $\bar{q}_{\widetilde{\omega}}=1$. For $n$ sufficiently large, we have that $U^{b}\left(\left\{\left(\omega, p_{\omega}^{n}\right)\right\} ; r^{n}\right)<U^{b}\left(\left\{\left(\widetilde{\omega}, p_{\widetilde{\omega}}^{n}\right)\right\} ; r^{n}\right)$, contradicting that $\left(p^{n}, q^{n}, A^{n}\right)$ is a QCCE of $\mathcal{E}^{n}$.

Thus Step 1 holds.

Step 2: Condition (ii) of Definition 3.6 holds.

By contradiction, suppose that there is $s \in S$ such that $\bar{A}^{s} \notin \delta^{s}(\bar{p})$.

In case $\bar{A}^{s}=\emptyset$, there is $\omega^{\prime} \in \Omega^{s}$ such that $U^{s}\left(\bar{A}^{s}\right)<U^{s}\left(\left\{\left(\omega^{\prime}, \bar{p}_{\omega^{\prime}}\right)\right\}\right)$. For $n$ sufficiently large, we have that $U^{s}(\emptyset)=U^{s}\left(A^{n}\right)<U^{s}\left(\left\{\left(\omega^{\prime}, p_{\omega^{\prime}}^{n}\right)\right\}\right)$, contradicting that $\left(p^{n}, q^{n}, A^{n}\right)$ is a QCCE of $\mathcal{E}^{n}$.

In case $\bar{A}^{s} \neq \emptyset$, let $\omega$ be the unique element of $\omega\left(\bar{A}^{s}\right)$. Then there is $\widetilde{A}^{s} \in \delta^{s}(\bar{p})$ such that $U^{s}\left(\bar{A}^{s}\right)<U^{s}\left(\widetilde{A}^{s}\right)$. If $\widetilde{A}^{s}=\emptyset$, we have for $n$ sufficiently large that $U^{s}\left(\left\{\omega, p_{\omega}^{n}\right\}\right)=$ $U^{s}\left(A^{n}\right)<U^{s}(\emptyset)$, contradicting that $\left(p^{n}, q^{n}, A^{n}\right)$ is a QCCE of $\mathcal{E}^{n}$. If $\widetilde{A}^{s} \neq \emptyset$, let $\widetilde{\omega}$ be the unique element of $\omega\left(\widetilde{A}^{s}\right)$. For $n$ sufficiently large, we have that $U^{s}\left(\left\{\omega, p_{\omega}^{n}\right\}\right)=U^{s}\left(A^{n}\right)<$ $U^{s}\left(\left\{\left(\widetilde{\omega}, p_{\widetilde{\omega}}^{n}\right)\right\}\right)$, contradicting that $\left(p^{n}, q^{n}, A^{n}\right)$ is a QCCE of $\mathcal{E}^{n}$.

Step 3: Condition (iii) of Definition 3.6 holds.

This follows immediately from the construction of $\bar{q}$.

Consequently, $(\bar{p}, \bar{q}, \bar{A})$ is a QCCE of $\overline{\mathcal{E}}$. 
Theorem 3.9 considers sequences $\left(p^{n}, A^{n}\right)_{n \in \mathbb{N}}$ of prices and outcomes and leaves out the quantity constraints $q^{n}$. Indeed, the limit of any convergent sequence of QCCEs of $\mathcal{E}^{n}$ may not be a QCCE of the limit economy. For instance, a sequence of competitive equilibria $\left(p^{n}, A^{n}\right)$ in Example 3.4 is a sequence of QCCEs $\left(p^{n}, q^{n}, A^{n}\right)$ with $q^{n}=(1, \ldots, 1)$. However, at the limit economy shown in Example 3.5, there is no competitive equilibrium so there is no QCCE that is compatible with quantity constraints $\bar{q}=(1, \ldots, 1)$.

In addition, not every set of QCCE trades at the limit economy corresponds to the limit of competitive equilibrium trades in economies with increasingly stringent liquidity constraints. In Example 3.7, $\left(\bar{p}, q^{\prime}, A^{\prime}\right)$ is a QCCE with set of equilibrium trades equal to $\left\{\omega_{31}, \omega_{22}\right\}$. However, $\left\{\omega_{31}, \omega_{22}\right\}$ is not compatible with any competitive equilibrium in an economy with soft liquidity constraints in Example 3.4.

A particularly interesting special case of Theorem 3.9 results when $\left(\mathcal{E}^{n}\right)_{n \in \mathbb{N}}$ is a sequence of economies with soft liquidity constraints that converges to a limit economy $\overline{\mathcal{E}}$ where at least one buyer is subject to a hard liquidity constraint. In such a case, $\left(p^{n}, A^{n}\right)_{n \in \mathbb{N}}$ is a sequence of competitive equilibria. Theorem 3.9 says that $\left(p^{n}, A^{n}\right)_{n \in \mathbb{N}}$ contains a convergent subsequence whose limit $(\bar{p}, \bar{A})$ is compatible with QCCE prices and outcome in the limit economy $\overline{\mathcal{E}}$. For instance, the limit of a sequence of competitive equilibria $\left(p^{n}, A^{n}\right)$ in Example 3.4 for increasing interest rates $\rho^{n}$ yields prices $\bar{p}$ and outcome $\bar{A}$ corresponding to the QCCE $(\bar{p}, \bar{q}, \bar{A})$ at the limit economy in Example 3.7.

Finally, even if a competitive equilibrium exists in an economy $\mathcal{E}$ with hard liquidity constraints and $\left(p^{n}, A^{n}\right)_{n \in \mathbb{N}}$ is a convergent sequence of competitive equilibria in this economy, then its limit $(\bar{p}, \bar{A})$ may not be a competitive equilibrium in the economy $\mathcal{E}$. Example 3.10 illustrates this point.

Example 3.10: Take $B=\left\{b_{1}, b_{2}\right\}, S=\left\{s_{1}\right\}$, and $\Omega=\left\{\omega_{11}, \omega_{21}\right\}$, where buyer $b_{1}$ can trade $\omega_{11}$ with $s_{1}$, and buyer $b_{2}$ can trade $\omega_{21}$ with $s_{1}$. All agents have utility functions as described in Example 2.1. For every agent $i \in B \cup S, u^{i}\left(o^{i}\right)=0$. Let $M^{b_{1}}=2, M^{b_{2}}=10$, $V^{b_{1}}\left(\omega_{11}\right)=5$, and $V^{b_{2}}\left(\omega_{21}\right)=4$. Let $r^{b_{1}}=+\infty$ and $r^{b_{2}}=0$.

For every $n \in \mathbb{N}$, let $\left(p^{n}, A^{n}\right) \in \mathbb{R}^{\Omega} \times \mathcal{A}$ be such that

$$
p_{\omega_{11}}^{n}=p_{\omega_{21}}^{n}=4-\frac{2 n}{1+n} \text { and } A^{n}=\left\{\left(\omega_{21}, p_{\omega_{21}}^{n}\right)\right\}
$$

It is easily seen that, for every $n \in \mathbb{N},\left(p^{n}, A^{n}\right)$ is a competitive equilibrium in this economy. At the limit when $n \rightarrow+\infty$, we have that $\bar{p}_{\omega_{11}}=\bar{p}_{\omega_{21}}=2$ and $\bar{A}=\left\{\left(\omega_{21}, \bar{p}_{\omega_{21}}\right)\right\}$. However, $(\bar{p}, \bar{A})$ is no longer a competitive equilibrium as the no-trade option is no longer part of the demand set of agent 1 .

In contrast, in an economy with soft liquidity constraints, the limit of a convergent sequence of competitive equilibria is a competitive equilibrium as well. 


\section{Expectational Equilibrium}

Herings (2020) proposes the notion of expectational equilibrium. An expectational equilibrium does not explicitly depend on prices but rather on expectations related to tradable contracts. The concept unifies all the existing approaches of competitive equilibrium that have been proposed in the literature so far and, in particular, can be applied to both settings with and settings without monetary transfers.

Let $\bar{Y}=\Omega \times \mathbb{R}$ be the set of all possible contracts and $2^{\bar{Y}}$ be the power set of $\bar{Y}$. Let $Q \subseteq \bar{Y}$ denote a set of rationing constraints on the buyers side. For every $b \in B, Q^{b}$ represents the set of contracts for which buyer $b$ expects no supply from the sellers side. Similarly, Let $R \subseteq \bar{Y}$ denote a set of rationing constraints on the sellers side. For every $s \in S, R^{s}$ is the set of contracts for which seller $s$ expects no demand from the buyers side. An expectational equilibrium corresponds to an endogenously determined profile of rationing constraints $(Q, R)$ together with an outcome $A$.

Given a set of rationing constraints $Q$, the rationed budget set of buyer $b \in B$ is given by

$$
\gamma^{b}(Q)=\left\{A^{b} \in X^{b} \mid A^{b} \cap Q^{b}=\emptyset\right\}
$$

and the rationed demand set of buyer $b \in B$ is given by

$$
\delta^{b}(Q ; r)=\underset{A^{b} \in \gamma^{b}(Q)}{\arg \max } U^{b}\left(A^{b} ; r\right) .
$$

The rationed budget set of buyer $b$ consists of all contracts for which the buyer does not expect rationing together with the no-trade option. The rationed demand set of buyer $b$ collects all choices that maximize buyer b's utility over the rationed budget set.

Similarly, given a set of rationing constraints $R$, the rationed budget set of seller $s \in S$ is given by

$$
\gamma^{s}(R)=\left\{A^{s} \in X^{s} \mid A^{s} \cap R^{s}=\emptyset\right\}
$$

and the rationed demand set of seller $s \in S$ is given by

$$
\delta^{s}(R)=\underset{A^{s} \in \gamma^{s}(R)}{\arg \max } U^{s}\left(A^{s}\right) .
$$

The definition of an expectational equilibrium is as follows.

Definition 4.1: An element $(A, Q, R) \in \mathbb{R}^{\Omega} \times 2^{\bar{Y}} \times 2^{\bar{Y}}$ is an expectational equilibrium for the economy $\mathcal{E}=(B, S, \Omega, u, M, r)$ if:

(i) For every $b \in B, A^{b} \in \delta^{b}(Q ; r)$. 
(ii) For every $s \in S, A^{s} \in \delta^{s}(R)$.

(iii) $Q \cap R=\emptyset$.

The first two conditions correspond to optimization by buyers and sellers given the profile of rationing constraints. A buyer $b \in B$ demands the best contract outside $Q^{b}$ and a seller $s \in S$ chooses the best contract outside $R^{s}$. The third condition expresses that markets are transparent. For a given contract, it cannot be the case that both sides of the market expect to be rationed at the same time.

Example 4.2 illustrates the notion of expectational equilibrium for the economy with soft liquidity constraints introduced in Example 3.4.

Example 4.2: Consider the economy with soft liquidity constraints in Example 3.4. Let $(A, Q, R) \in \mathbb{R}^{\Omega} \times 2^{\bar{Y}} \times 2^{\bar{Y}}$ be defined by

$$
\begin{aligned}
& A=\left\{\left(\omega_{11}, \frac{3+2 \rho}{1+\rho}\right),\left(\omega_{32}, \frac{4+3 \rho}{1+\rho}\right)\right\}, \\
& Q=\left\{(\omega, t) \in \bar{Y} \mid \omega \in\left\{\omega_{11}, \omega_{31}\right\}, t<\frac{3+2 \rho}{1+\rho}\right\} \cup\left\{(\omega, t) \in \bar{Y} \mid \omega \in\left\{\omega_{22}, \omega_{32}\right\}, t<\frac{4+3 \rho}{1+\rho}\right\}, \\
& R=\left\{(\omega, t) \in \bar{Y} \mid \omega \in\left\{\omega_{11}, \omega_{31}\right\}, t>\frac{3+2 \rho}{1+\rho}\right\} \cup\left\{(\omega, t) \in \bar{Y} \mid \omega \in\left\{\omega_{22}, \omega_{32}\right\}, t>\frac{4+3 \rho}{1+\rho}\right\} .
\end{aligned}
$$

We show that $(A, Q, R)$ is an expectational equilibrium by verifying Conditions (i), (ii), and (iii) of Definition 4.1.

First, $(A, Q, R)$ satisfies Condition (i). For buyer $b_{1}$ we have

$$
\begin{aligned}
\gamma^{b_{1}}(Q) & =\left\{\left\{\left(\omega_{11}, t\right)\right\} \in X^{b_{1}} \mid t \geq \frac{3+2 \rho}{1+\rho}\right\} \cup\{\emptyset\} \\
A^{b_{1}} & =\left\{\left(\omega_{11}, \frac{3+2 \rho}{1+\rho}\right)\right\} \in \delta^{b_{1}}(Q ; \rho)=\left\{\left\{\left(\omega_{11}, \frac{3+2 \rho}{1+\rho}\right)\right\}\right\} .
\end{aligned}
$$

For buyer $b_{2}$ it holds that

$$
\begin{aligned}
\gamma^{b_{2}}(Q) & =\left\{\left\{\left(\omega_{22}, t\right)\right\} \in X^{b_{2}} \mid t \geq \frac{4+3 \rho}{1+\rho}\right\} \cup\{\emptyset\}, \\
A^{b_{2}} & =\emptyset \in \delta^{b_{2}}(Q ; \rho)=\left\{\left\{\left(\omega_{22}, \frac{4+3 \rho}{1+\rho}\right)\right\}, \emptyset\right\} .
\end{aligned}
$$

For buyer $b_{3}$ it holds that

$$
\begin{aligned}
\gamma^{b_{3}}(Q) & =\left\{\left\{\left(\omega_{31}, t\right)\right\} \in X^{b_{3}} \mid t \geq \frac{3+2 \rho}{1+\rho}\right\} \cup\left\{\left\{\left(\omega_{32}, t\right)\right\} \in X^{b_{3}} \mid t \geq \frac{4+3 \rho}{1+\rho}\right\} \cup\{\emptyset\}, \\
A^{b_{3}} & =\left\{\left(\omega_{32}, \frac{4+3 \rho}{1+\rho}\right)\right\} \in \delta^{b_{3}}(Q ; \rho)=\left\{\left\{\left(\omega_{31}, \frac{3+2 \rho}{1+\rho}\right)\right\},\left\{\left(\omega_{32}, \frac{4+3 \rho}{1+\rho}\right)\right\}\right\} .
\end{aligned}
$$

Second, $(A, Q, R)$ satisfies Condition (ii). For seller $s_{1}$ we have

$$
\begin{aligned}
\gamma^{s_{1}}(R) & =\left\{\{(\omega, t)\} \in X^{s_{1}} \mid \omega \in\left\{\omega_{11}, \omega_{31}\right\}, t \leq \frac{3+2 \rho}{1+\rho}\right\} \cup\{\emptyset\}, \\
A^{s_{1}} & =\left\{\left(\omega_{11}, \frac{3+2 \rho}{1+\rho}\right)\right\} \in \delta^{s_{1}}(R)=\left\{\left\{\left(\omega_{11}, \frac{3+2 \rho}{1+\rho}\right)\right\},\left\{\left(\omega_{31}, \frac{3+2 \rho}{1+\rho}\right)\right\}\right\} .
\end{aligned}
$$

For seller $s_{2}$ it holds that

$$
\begin{aligned}
\gamma^{s_{2}}(R) & =\left\{\{(\omega, t)\} \in X^{s_{2}} \mid \omega \in\left\{\omega_{22}, \omega_{32}\right\}, t \leq \frac{4+3 \rho}{1+\rho}\right\} \cup\{\emptyset\}, \\
A^{s_{2}} & =\left\{\left(\omega_{32}, \frac{4+3 \rho}{1+\rho}\right)\right\} \in \delta^{s_{2}}(R)=\left\{\left\{\left(\omega_{22}, \frac{4+3 \rho}{1+\rho}\right)\right\},\left\{\left(\omega_{32}, \frac{4+3 \rho}{1+\rho}\right)\right\}\right\} .
\end{aligned}
$$


It is easily verified that Condition (iii) holds.

Both the model with soft and the model with hard liquidity constraints satisfy the assumptions of Theorem 5.5 of Herings (2020). We therefore obtain the following result.

Proposition 4.3: Let $\mathcal{E}=(B, S, \Omega, u, M, r)$ be an economy such that $r \in[0,+\infty]^{B}$. An expectational equilibrium exists.

Furthermore, the following limit result holds for expectational equilibrium.

Theorem 4.4: Let $\left(\mathcal{E}^{n}\right)_{n \in \mathbb{N}}$ be a sequence of economies that converges to an economy $\overline{\mathcal{E}}$. For every $n \in \mathbb{N}$, let $\left(A^{n}, Q^{n}, R^{n}\right)$ be an expectational equilibrium of the economy $\mathcal{E}^{n}$.

(i) The sequence $\left(A^{n}\right)_{n \in \mathbb{N}}$ has a convergent subsequence.

(ii) Let $\bar{A}$ be the limit of a convergent subsequence of $\left(A^{n}\right)_{n \in \mathbb{N}}$. Then there is $(\bar{Q}, \bar{R}) \in$ $2^{\bar{Y}} \times 2^{\bar{Y}}$ such that $(\bar{A}, \bar{Q}, \bar{R})$ is an expectational equilibrium of $\overline{\mathcal{E}}$.

\section{Proof:}

Part (i): Since the set of trades is finite, there is a subsequence $\left(A^{n_{m}}\right)_{m \in \mathbb{N}}$ of $\left(A^{n}\right)_{n \in \mathbb{N}}$ such that the set of trades $\omega\left(A^{n_{m}}\right)$ does not depend on $n_{m}$. Define $\bar{\Omega}=\omega\left(A^{n_{m}}\right)$.

Let $\omega \in \bar{\Omega}, b=b(\omega)$, and $s=s(\omega)$. For every $m \in \mathbb{N}$, let $t^{n_{m}} \in \mathbb{R}$ be such that $\left(\omega, t^{n_{m}}\right) \in A^{n_{m}}$. We show that the sequence $\left(t^{n_{m}}\right)_{m \in \mathbb{N}}$ is bounded.

Since $A^{n_{m}}$ is an expectational equilibrium and $\emptyset \in \gamma^{s}\left(R^{n_{m}}\right)$, it holds that $u^{s}\left(\omega, t^{n_{m}}\right)=$ $U^{s}\left(\left\{\left(\omega, t^{n_{m}}\right)\right\}\right) \geq U^{s}(\emptyset)=u^{s}\left(o^{s}\right)=u^{s}\left(\omega, \underline{t}_{\omega}^{s}\right)$. It follows that $\underline{t}_{\omega}^{s} \leq t^{n_{m}}$.

There is $\widetilde{t}^{n_{m}} \geq t^{n_{m}}$ such that $u^{b}\left(\omega, \widetilde{t}^{n_{m}}\right)=U^{b}\left(\left\{\left(\omega, t^{n_{m}}\right)\right\} ; r^{n_{m}}\right)$. Since $A^{n_{m}}$ is an expectational equilibrium outcome and $\emptyset \in \gamma^{b}\left(Q^{n_{m}}\right)$, it holds that $U^{b}\left(\left\{\left(\omega, t^{n_{m}}\right)\right\} ; r^{n_{m}}\right) \geq u^{b}\left(o^{b}\right)=$ $u^{b}\left(\omega, \bar{t}_{\omega}^{b}\right)$, and so $u^{b}\left(\omega, \widetilde{t}^{n_{m}}\right) \geq u^{b}\left(\omega, \bar{t}_{\omega}^{b}\right)$. It follows that $t^{n_{m}} \leq \widetilde{t}^{n_{m}} \leq \bar{t}_{\omega}^{b}$.

We conclude that, for every $m \in \mathbb{N}, \underline{t}_{\omega}^{s} \leq t^{n_{m}} \leq \bar{t}_{\omega}^{b}$.

It follows that the sequence $\left(A^{n}\right)_{n \in \mathbb{N}}$ has a convergent subsequence.

Part (ii): Without loss of generality, assume the sequence $\left(A^{n}\right)_{n \in \mathbb{N}}$ is convergent and has limit $\bar{A}$. Define $\bar{\Omega}=\omega(\bar{A})$ and, for every $c \in \bar{A}, \bar{t}(c)=t(c)$. We construct $(\bar{Q}, \bar{R}) \in 2^{\bar{Y}} \times 2^{\bar{Y}}$ such that $(\bar{A}, \bar{Q}, \bar{R})$ is an expectational equilibrium of $\overline{\mathcal{E}}$.

For every $s \in S$, for every $\omega \in \Omega^{s}$, there is $t_{\omega}^{s} \in \mathbb{R}$ such that $u^{s}\left(\omega, t_{\omega}^{s}\right)=U^{s}(\bar{A})$. In case $c \in \bar{A}^{s}$, it holds that $t_{\omega(c)}^{s}=\bar{t}(c)$.

Step 1: Let $\omega \in \Omega \backslash \bar{\Omega}, b=b(\omega)$, and $s=s(\omega)$. If $U^{b}(\bar{A} ; \bar{r})<U^{b}\left(\left\{\left(\omega, t_{\omega}^{s}\right)\right\} ; \bar{r}\right)$, then $\bar{r}^{b}=+\infty$ and $t_{\omega}^{s}=M^{b}$.

Let $U^{b}(\bar{A} ; \bar{r})<U^{b}\left(\left\{\left(\omega, t_{\omega}^{s}\right)\right\} ; \bar{r}\right)$. We show first that $\bar{r}^{b}=+\infty$. By contradiction, suppose that $\bar{r}^{b}<+\infty$. Since $U^{b}(\bar{A} ; \bar{r})<U^{b}\left(\left\{\left(\omega, t_{\omega}^{s}\right)\right\} ; \bar{r}\right)$ and $\bar{r}^{b}<+\infty$, for sufficiently large $n$, there is $t_{\omega}^{n, b} \in \mathbb{R}$ and $t_{\omega}^{n, s} \in \mathbb{R}$ such that $u^{b}\left(\omega, t_{\omega}^{n, b}\right)=U^{b}\left(A^{n} ; r^{n}\right), u^{s}\left(\omega, t_{\omega}^{n, s}\right)=$ 
$U^{s}\left(A^{n}\right)$, and $t_{\omega}^{n, b}>t_{\omega}^{n, s}$. Thus, there is $t^{n} \in\left(t_{\omega}^{n, s}, t_{\omega}^{n, b}\right)$ such that $U^{b}\left(\left\{\left(\omega, t^{n}\right)\right\} ; r^{n}\right)>$ $U^{b}\left(A^{n} ; r^{n}\right)$ and $u^{s}\left(\omega, t^{n}\right)>U^{s}\left(A^{n}\right)$. Since $A^{n}$ is an expectational equilibrium outcome, there is $\left(Q^{n}, R^{n}\right) \in 2^{\bar{Y}} \times 2^{\bar{Y}}$ such that $\left(A^{n}, Q^{n}, R^{n}\right)$ is an expectational equilibrium of $\mathcal{E}^{n}$. Since $U^{b}\left(\left\{\left(\omega, t^{n}\right)\right\} ; r^{n}\right)>U^{b}\left(A^{n} ; r^{n}\right)$ and $u^{s}\left(\omega, t^{n}\right)>U^{s}\left(A^{n}\right)$, by Conditions (i) and (ii) of Definition 4.1, we have that $\left(\omega, t^{n}\right) \in Q^{n}$ and $\left(\omega, t^{n}\right) \in R^{n}$, contradicting Condition (iii) of Definition 4.1. Consequently, it holds that $\bar{r}^{b}=+\infty$.

Second we show that $t_{\omega}^{s}=M^{b}$. By contradiction suppose not, i.e., $t_{\omega}^{s} \neq M^{b}$. If $t_{\omega}^{s}>M^{b}$, we have that $U^{b}\left(\left\{\left(\omega, t_{\omega}^{s}\right)\right\} ; \bar{r}\right)=-\infty<U^{b}(\bar{A} ; \bar{r})$, contradicting $U^{b}(\bar{A} ; \bar{r})<U^{b}\left(\left\{\left(\omega, t_{\omega}^{s}\right)\right\} ; \bar{r}\right)$. Thus, it holds that $t_{\omega}^{s}<M^{b}$. Thus, for sufficiently large $n$, there is $t^{n} \in\left(t_{\omega}^{s}, M^{b}\right)$ such that $U^{b}\left(\left\{\left(\omega, t^{n}\right)\right\} ; r^{n}\right)>U^{b}\left(A^{n} ; r^{n}\right)$ and $u^{s}\left(\omega, t^{n}\right)>U^{s}\left(A^{n}\right)$. Since $A^{n}$ is an expectational equilibrium outcome, there is $\left(Q^{n}, R^{n}\right) \in 2^{\bar{Y}} \times 2^{\bar{Y}}$ such that $\left(A^{n}, Q^{n}, R^{n}\right)$ is an expectational equilibrium of $\mathcal{E}^{n}$. Since $U^{b}\left(\left\{\left(\omega, t^{n}\right)\right\} ; r^{n}\right)>U^{b}\left(A^{n} ; r^{n}\right)$ and $u^{s}\left(\omega, t^{n}\right)>U^{s}\left(A^{n}\right)$, by Conditions (i) and (ii) of Definition 4.1, we have that $\left(\omega, t^{n}\right) \in Q^{n}$ and $\left(\omega, t^{n}\right) \in R^{n}$, contradicting Condition (iii) of Definition 4.1. Consequently, it holds that $t_{\omega}^{s}=M^{b}$.

Step 2: Construction of $(\bar{Q}, \bar{R})$.

For every $b \in B$, we define

$$
\bar{Q}^{b}=\left\{(\omega, t) \in \Omega^{b} \times \mathbb{R} \mid \omega \in \bar{\Omega} \& t<t_{\omega}^{s(\omega)}\right\} \cup\left\{(\omega, t) \in \Omega^{b} \times \mathbb{R} \mid \omega \in \Omega \backslash \bar{\Omega} \& t \leq t_{\omega}^{s(\omega)}\right\} .
$$

For every $s \in S$, we define

$$
\bar{R}^{s}=\left\{(\omega, t) \in \Omega^{s} \times \mathbb{R} \mid t>t_{\omega}^{s}\right\} .
$$

Step 3: $(\bar{A}, \bar{Q}, \bar{R})$ is an expectational equilibrium.

Let $b \in B$. By the construction of $\bar{Q}^{b}$, we have

$$
\gamma^{b}(\bar{Q})=\left\{\{(\omega, t)\} \in X^{b} \mid \omega \in \bar{\Omega} \& t \geq t_{\omega}^{s(\omega)}\right\} \cup\left\{\{(\omega, t)\} \in X^{b} \mid \omega \in \Omega \backslash \bar{\Omega} \& t>t_{\omega}^{s(\omega)}\right\} \cup\{\emptyset\} .
$$

Let $(\omega, t) \in \Omega^{b} \times \mathbb{R}$ be such that $\omega \in \bar{\Omega}$. For every $t \geq t_{\omega}^{s(\omega)}$, it holds that $U^{b}\left(\bar{A}^{b} ; \bar{r}\right) \geq$ $U^{b}(\{(\omega, t)\} ; \bar{r})$. Let $(\omega, t) \in \Omega^{b} \times \mathbb{R}$ be such that $\omega \in \Omega^{b} \backslash \bar{\Omega}$, and let $s=s(\omega)$. If $U^{b}(\bar{A} ; \bar{r})<$ $U^{b}\left(\left\{\left(\omega, t_{\omega}^{s}\right)\right\} ; \bar{r}\right)$, by Step 1 , it means that $\bar{r}^{b}=+\infty$ and $t_{\omega}^{s}=M^{b}$. Thus, for every $t>t_{\omega}^{s}$, it holds that $U^{b}(\bar{A} ; \bar{r})>U^{b}(\{(\omega, t)\} ; \bar{r})$. If $U^{b}(\bar{A} ; \bar{r}) \geq U^{b}\left(\left\{\left(\omega, t_{\omega}^{s}\right)\right\} ; \bar{r}\right)$, for every $t>t_{\omega}^{s}$, it holds that $U^{b}(\bar{A} ; \bar{r}) \geq U^{b}(\{(\omega, t)\} ; \bar{r})$. It is easy to see that $U^{b}(\bar{A} ; \bar{r}) \geq U^{b}(\emptyset ; \bar{r})$. Thus Condition (i) of Definition 4.1 holds.

Let $s \in S$. By the construction of $\bar{R}^{s}, \gamma^{s}(\bar{R})=\left\{\{(\omega, t)\} \in X^{s} \mid t \leq t_{\omega}^{s}\right\} \cup\{\emptyset\}$. It holds that $U^{s}\left(\bar{A}^{s}\right)=U^{s}\left(\left\{\left(\omega, t_{\omega}^{s}\right)\right\}\right) \geq U^{s}(\{(\omega, t)\})$ for every $(\omega, t) \in \Omega^{s} \times \mathbb{R}$ such that $t \leq t_{\omega}^{s}$. It is easy to see that $U^{s}(\bar{A}) \geq U^{s}(\emptyset)$. Thus Condition (ii) of Definition 4.1 holds.

By Step 2, it holds that $\bar{Q} \cap \bar{R}=\emptyset$. Thus Condition (iii) of Definition 4.1 holds. Q.E.D. 
Theorem 4.4 implies that expectational equilibrium does not suffer from discontinuity problems in case a sequence of economies with increasingly stringent soft liquidity constraints converges to a limit economy with hard liquidity constraints. For illustration, consider a sequence of expectational equilibrium outcomes $\left(A^{n}\right)_{n \in \mathbb{N}}$ in Example 4.2 with increasing interest rates $\rho^{n}$ that go to $+\infty$. Its limit is equal to $\bar{A}=\left\{\left(\omega_{11}, 2\right),\left(\omega_{32}, 3\right)\right\}$. We define $\bar{Q}=\left\{(\omega, t) \in \bar{Y} \mid \omega \in\left\{\omega_{11}, \omega_{31}\right\}, t<2\right\} \cup\left\{(\omega, t) \in \bar{Y} \mid \omega \in\left\{\omega_{22}, \omega_{32}\right\}, t<\right.$ $3\} \cup\left\{\left(\omega_{22}, 3\right)\right\}$ and $\bar{R}=\left\{(\omega, t) \in \bar{Y} \mid \omega \in\left\{\omega_{11}, \omega_{31}\right\}, t>2\right\} \cup\{(\omega, t) \in \bar{Y} \mid \omega \in$ $\left.\left\{\omega_{22}, \omega_{32}\right\}, t>3\right\}$ and observe that $(\bar{A}, \bar{Q}, \bar{R})$ is an expectational equilibrium of the limit economy $\overline{\mathcal{E}}$. Notice that $A^{\prime}=\left\{\left(\omega_{31}, 2\right),\left(\omega_{22}, 3\right)\right\}$ is also an expectational equilibrium outcome at the limit economy, but is not compatible with any convergent sequence of expectational equilibrium outcomes at the economy with soft liquidity constraints in Example 4.2.

\section{Discussion}

In this section, we first establish a novel equivalence between QCCE outcomes and expectational equilibrium outcomes. Then we show that both solution concepts coincide with those coming from cooperative game theory like stable outcome and core outcome. This implies that also stable outcomes and core outcomes do not suffer from discontinuity problems like competitive equilibrium.

We first establish the following equivalence result.

Theorem 5.1: Let $\mathcal{E}=(B, S, \Omega, u, M, r)$ be an economy such that $r \in[0,+\infty]^{B}$. The sets of QCCE outcomes and expectational equilibrium outcomes coincide.

Proof: The proof consists of the following two steps.

Step 1: If $(p, q, A)$ is a QCCE of $\mathcal{E}$, there is $(Q, R) \in 2^{\bar{Y}} \times 2^{\bar{Y}}$ such that $(A, Q, R)$ is an expectational equilibrium.

For every $b \in B$, we define

$$
Q^{b}=\left\{(\omega, t) \in \Omega^{b} \times \mathbb{R} \mid \omega \in \omega(A) \& t<p_{\omega}\right\} \cup\left\{(\omega, t) \in \Omega^{b} \times \mathbb{R} \mid \omega \in \Omega \backslash \omega(A) \& t \leq p_{\omega}\right\} .
$$

For every $s \in S$, we define

$$
R^{s}=\left\{(\omega, t) \in \Omega^{s} \times \mathbb{R} \mid t>p_{\omega}\right\} .
$$

By construction, we have that $Q \cap R=\emptyset$ and so Condition (iii) of Definition 4.1 holds for $(A, Q, R)$. We show below that Conditions (i) and (ii) of Definition 4.1 hold for $(A, Q, R)$.

Let $b \in B$. By the construction of $Q^{b}$, we have

$\gamma^{b}(Q)=\left\{\{(\omega, t)\} \in X^{b} \mid \omega \in \omega(A) \& t \geq p_{\omega}\right\} \cup\left\{\{(\omega, t)\} \in X^{b} \mid \omega \in \Omega \backslash \omega(A) \& t>p_{\omega}\right\} \cup\{\emptyset\}$. 
By Condition (i) of Definition 3.6, we have that $U^{b}\left(A^{b} ; r\right) \geq U^{b}(\emptyset)$. Let $\{c\} \in \gamma^{b}(Q)$ be such that $\omega(c) \in \Omega \backslash \omega(A)$. In case $q_{\omega(c)}=1$, by Condition (i) of Definition 3.6, it holds that $U^{b}\left(A^{b} ; r\right) \geq U^{b}\left(\left\{\left(\omega, p_{\omega}\right)\right\} ; r\right) \geq U^{b}(\{c\} ; r)$. In case $q_{\omega(c)}=0$, by Condition (iii) of Definition 3.6 , it holds that $r^{b}=+\infty$ and $t(c)>p_{\omega(c)} \geq M^{b}$. Thus $U^{b}\left(A^{b} ; r\right)>U^{b}(\{c\} ; r)$. If $A^{b}=\emptyset$, we are done. If $A^{b}=\left\{\left(\omega^{\prime}, p_{\omega^{\prime}}\right)\right\}$, it holds that $U^{b}(A ; r) \geq U^{b}\left(\left\{\left(\omega^{\prime}, t^{\prime}\right)\right\} ; r\right)$ for every $t^{\prime} \geq p_{\omega^{\prime}}$. Consequently, Condition (i) of Definition 4.1 holds.

Let $s \in S$. By the construction of $R^{s}, \gamma^{s}(R)=\left\{\{(\omega, t)\} \in X^{s} \mid t \leq p_{\omega}\right\} \cup\{\emptyset\}$. Since $(p, q, A)$ is a QCCE, by Condition (ii) of Definition 3.6, we have that $U^{s}(A) \geq U^{s}(\emptyset)$, and for every $\{(\omega, t)\} \in \gamma^{s}(R)$, it holds that $U^{s}(A) \geq u^{s}\left(\omega, p_{\omega}\right) \geq u^{s}(\omega, t)$. Thus Condition (ii) of Definition 4.1 holds.

Step 2: If $(A, Q, R)$ is an expectational equilibrium of $\mathcal{E}$, there is $(p, q) \in \mathbb{R}^{\Omega} \times\{0,1\}^{\Omega}$ such that $(p, q, A)$ is a QCCE of $\mathcal{E}$.

For every $s \in S$, for every $\omega \in \Omega^{s}$, there is $p_{\omega} \in \mathbb{R}$ such that $u^{s}(A)=u^{s}\left(\omega, p_{\omega}\right)$. Let $p_{\omega} \in \mathbb{R}$ be the price of trade $\omega$. For every $\omega \in \Omega$, if $r^{b(\omega)}=+\infty$ and $p_{\omega} \geq M^{b(\omega)}, q_{\omega}=0$, and if $r^{b(\omega)}<+\infty$ or $p_{\omega}<M^{b(\omega)}, q_{\omega}=1$. By the construction of $(p, q) \in \mathbb{R}^{\Omega} \times\{0,1\}^{\Omega}$, it is easily seen that Conditions (ii) and (iii) of Definition 3.6 hold for $(p, q, A)$. We show next that Condition (i) of Definition 3.6 holds for $(p, q, A)$.

By contradiction, suppose Condition (i) of Definition 3.6 fails to hold. Then there is $b \in B$ such that $A^{b} \notin \delta^{b}(p, q ; r)$. Since $(A, Q, R)$ is an expectational equilibrium, by Condition (i) of Definition 4.1, $U^{b}\left(A^{b} ; r\right) \geq U^{b}(\emptyset)$ and so there is $\left\{\left(\omega, p_{\omega}\right)\right\} \in \delta^{b}(p, q ; r)$ such that $U^{b}\left(A^{b} ; r\right)<U^{b}\left(\left\{\left(\omega, p_{\omega}\right)\right\} ; r\right)$ and $q_{\omega}=1$. By the construction of $q$, either $r^{b(\omega)}<+\infty$ or $p_{\omega}<M^{b(\omega)}$ holds. In either case, there is $t>p_{\omega}$ such that $U^{b}\left(A^{b} ; r\right)<U^{b}(\{(\omega, t)\} ; r)$ and $U^{s(\omega)}(A)=U^{s(\omega)}\left(\left\{\left(\omega, p_{\omega}\right)\right\}\right)<U^{s(\omega)}(\{(\omega, t)\})$. Since $(A, Q, R)$ is an expectational equilibrium, by Conditions (i) and (ii) of Definition 4.1, we have that $\{(\omega, t)\} \in Q^{b}$ and $\{(\omega, t)\} \in R^{s(\omega)}$, contradicting Condition (iii) of Definition 4.1.

Q.E.D.

In the following, we define stable outcome and core outcome.

For every $Y \subseteq \Omega \times \mathbb{R}$, the sets of optimal choices of buyer $b \in B$ and seller $s \in S$ within the set of contracts $Y$ are defined as

$$
\begin{aligned}
& C^{b}(Y)=\underset{\left\{A^{b} \in X^{b} \mid A^{b} \subseteq Y^{b}\right\}}{\arg \max } U^{b}\left(A^{b} ; r\right), \\
& C^{s}(Y)=\underset{\left\{A^{s} \in X^{s} \mid A^{s} \subseteq Y^{s}\right\}}{\arg \max } U^{s}\left(A^{s}\right) .
\end{aligned}
$$

Notice that for agent $i \in B \cup S, C^{i}(Y)=\{\emptyset\}$ means that the no-trade option is weakly preferred to any choice in $Y$.

Definition 5.2: An outcome $A \in \mathcal{A}$ is stable for the economy $\mathcal{E}=(B, S, \Omega, u, M, r)$ if:

(i) For every $i \in B \cup S, A^{i} \in C^{i}(A)$. 
(ii) There is no $c=(\omega, t) \in \bar{Y}$ such that $U^{b(\omega)}(\{c\} ; r)>U^{b(\omega)}(A ; r)$ and $U^{s(\omega)}(\{c\})>$ $U^{s(\omega)}(A)$.

A stable outcome is a set of contracts that contains at most one contract for each agent. Condition (i) of Definition 5.2 says that every agent involved in a contract prefers this contract weakly to the no-trade option. Condition (ii) of Definition 5.2 says that there are no two agents who can sign a contract that makes both of them strictly better off.

Definition 5.3: An outcome $A \in \mathcal{A}$ is a core outcome for the economy $\mathcal{E}=(B, S, \Omega, u, M, r)$ if there is no outcome $A^{\prime} \in \mathcal{A}$ and a set of agents $N \subseteq B \cup S$ such that:

(i) For every $i \in(B \cup S) \backslash N,\left(A^{\prime}\right)^{i}=\emptyset$.

(ii) For every $b \in N \cap B, U^{b}\left(A^{\prime} ; r\right)>U^{b}(A ; r)$.

(iii) For every $s \in N \cap S, U^{s}\left(A^{\prime}\right)>U^{s}(A)$.

An outcome is a core outcome if no coalition of agents can propose a better outcome satisfying the following two conditions: Agents outside the coalition obtain the no-trade option, i.e., Condition (i) of Definition 5.3, and each agent in the coalition is strictly better off, i.e., Condition (ii) and (iii) of Definition 5.3.

Herings and Zhou (2021) show the equivalence between QCCE outcomes, stable outcomes, and core outcomes. Therefore, together with Theorem 5.1, this leads to the following corollary.

Corollary 5.4: Let $\mathcal{E}=(B, S, \Omega, u, M, r)$ be an economy such that $r \in[0,+\infty]^{B}$. The sets of QCCE outcomes, expectational equilibrium outcomes, stable outcomes, and core outcomes coincide.

In our setting, the solution concepts mentioned in Corollary 5.4 coincide. Nevertheless, there is no general equivalence between stable outcomes and core outcomes, or core outcomes and expectational equilibrium outcomes, if different models are considered, see Herings (2020) for details.

Theorems 3.9 and 4.4 together with Corollary 5.4 imply the following limit result.

Corollary 5.5: Let $\left(\mathcal{E}^{n}\right)_{n \in \mathbb{N}}$ be a sequence of economies that converges to an economy $\overline{\mathcal{E}}$. For every $n \in \mathbb{N}$, let $A^{n}$ be a stable outcome (respectively a core outcome) of $\mathcal{E}^{n}$.

(i) The sequence $\left(A^{n}\right)_{n \in \mathbb{N}}$ has a convergent subsequence.

(ii) Let $\bar{A}$ be the limit of a convergent subsequence of $\left(A^{n}\right)_{n \in \mathbb{N}}$. Then $\bar{A}$ is a stable outcome (respectively core outcome) of $\overline{\mathcal{E}}$. 
The sets of "strongly stable outcomes," "strict core outcomes," and competitive equilibrium outcomes coincide in an economy with soft liquidity constraints (Crawford and Knoer, 1981). ${ }^{6}$ However, in an economy with hard liquidity constraints, the sets of strongly stable outcomes and strict core outcomes can be empty (Herings and Zhou, 2021). Thus, a convergent sequence of competitive equilibrium outcomes, strongly stable outcomes, or strict core outcomes in economies with increasingly stringent soft liquidity constraints may not be a competitive equilibrium outcome, strongly stable outcome or strict core outcome at the limit economy with hard liquidity constraints, respectively.

\section{Concluding remarks}

We study a matching with contracts model where buyers may face soft or hard liquidity constraints. The economy with hard constraints follows as the limit of a sequence of economies with increasingly stringent soft constraints. However, the limit of a sequence of competitive equilibria for economies with increasingly stringent soft liquidity constraints may fail to be a competitive equilibrium of the limit economy with hard liquidity constraints.

We show that two alternative notions of competitive equilibrium, quantity constrained competitive equilibrium (QCCE) and expectational equilibrium, do not suffer from such deficiencies. In addition, we show the equivalence between QCCE outcomes, expectational equilibrium outcomes, and solution concepts from cooperative game theory like the core and stable outcomes, and discuss some implications of the limit results obtained. Our results provide new insights for the continuity properties of equilibria in matching models with different types of liquidity constraints.

\section{References}

[1] Blalock, G., Gertler, P.J., Levine, D.I., 2008. Financial constraints on investment in an emerging market crisis. Journal of Monetary Economics 55(3), 568-591.

[2] Carroll, C.D., 2001. A theory of the consumption function, with and without liquidity constraints. Journal of Economic Perspectives 15(3), 23-45.

[3] Che, Y.K., Gale, I. 1998. Standard auctions with financially constrained bidders. Review of Economic Studies 65(1), 1-21.

\footnotetext{
${ }^{6}$ Formal definitions of strongly stable outcomes and strict core outcomes can be found in Crawford and Knoer (1981) or Herings and Zhou (2021).
} 
[4] Crawford, V.P., Knoer, E.M., 1981. Job matching with heterogeneous firms and workers. Econometrica 49(2), 437-450.

[5] Demange, G., Gale, D., 1985. The strategy structure of two-sided matching markets. Econometrica 53(4), 873-888.

[6] Fleiner, T., Jagadeesan, R., Jankó, Z., Teytelboym, A., 2019. Trading networks with frictions. Econometrica 87(5), 1633-1661.

[7] Galichon, A., Kominers, S.D., Weber, S., 2019. Costly concessions: An empirical framework for matching with imperfectly transferable utility. Journal of Political Economy 127(6), 2875-2925.

[8] Hatfield, J.W., Kominers, S.D., Nichifor, A., Ostrovsky, M., Westkamp, A., 2013. Stability and competitive equilibrium in trading networks. Journal of Political Economy 121(5), 966-1005.

[9] Hatfield, J.W., Milgrom, P.R., 2005. Matching with contracts. American Economic Review 95(4), 913-935.

[10] Herings, P.J.J., 2020. Expectational equilibria in many-to-one matching models with contracts-a reformulation of competitive equilibrium. Maastricht University, GSBE Research Memoranda, No. 018.

[11] Herings, P.J.J., Zhou, Y., 2021. Competitive equilibria in matching models with financial constraints. International Economic Review, forthcoming.

[12] Legros, P., Newman, A. F., 2007. Beauty is a beast, frog is a prince: Assortative matching with nontransferabilities. Econometrica 75(4), 1073-1102.

[13] Quinzii, M., 1984. Core and competitive equilibria with indivisibilities. International Journal of Game Theory 13(1), 41-60.

[14] Saitoh, H., Serizawa, S., 2008. Vickrey allocation rule with income effect. Economic Theory 35(2), 391-401.

[15] Schlegel, J.C., 2021. The structure of equilibria in trading networks with frictions. Theoretical Economics, forthcoming.

[16] Talman, A.J.J., Yang, Z., 2015. An efficient multi-item dynamic auction with budget constrained bidders. International Journal of Game Theory 44(3), 769-784. 